\title{
Checklist for the crop weeds of Paraguay
}

\author{
Juana De Egea ${ }^{1,2}$, Fátima Mereles ${ }^{1,2}$, María del Carmen Peña-Chocarro ${ }^{3}$, \\ Gloria Céspedes ${ }^{1,2,4}$
}

I Associate Researcher for the Centro para el Desarrollo de la Investigación Cientifica. Fundación Moisés Bertoni para la Conservación de la Naturaleza y Laboratorios Díaz-Gill, Manduvirá 635, Asunción, Paraguay 2 Researcher for the Programa Nacional de Incentivo a Investigadores del Consejo Nacional de Ciencia y Tecnologia (CONACYT), Paraguay. 3 The Natural History Museum, Cromwell Rd, London SW75BD, U.K. 4 Asociación Etnobotánica Paraguaya, Julia Miranda Cueto de Estigarribia 795, Edificio Mariucci, ${ }^{\circ}$ piso, oficina 208, San Lorenzo, Paraguay

Corresponding author: Juana De Egea (juana.deegea@gmail.com)

Academic editor: A. Machado | Received 10 August 2016 | Accepted 7 October 2016 | Published 18 October 2016

Citation: De Egea J, Mereles F, Peña-Chocarro MC, Céspedes G (2016) Checklist for the crop weeds of Paraguay. PhytoKeys 73: 13-92. doi: 10.3897/phytokeys.73.10135

\begin{abstract}
Paraguay, a country whose economy is based mainly on agriculture and livestock for export, has experienced a major expansion in mechanized crops during the last few decades. Despite being heavily dependent on agriculture, Paraguay has very limited research on crop weeds, in spite of these having a high economic impact on production. This work aims to update and enhance the knowledgebase on the most common weeds affecting productive fields throughout the different ecoregions of Paraguay. We present here the first checklist of crop weeds for the country, which includes a total of 256 taxa (189 species, 10 subspecies, 54 varieties and 3 forms), with the most species-rich families being Poaceae and Asteraceae followed by Malvaceae, Amaranthaceae, Fabaceae and Solanaceae. The list includes three new records for the country. Synonyms, distribution details within Paraguay, habit and a voucher specimen are provided for each taxon.
\end{abstract}

\section{Keywords}

Weeds, crops, Paraguay, agro-ecosystems, new records

Copyright Juana De Egea et al. This is an open access article distributed under the terms of the Creative Commons Attribution License (CC BY 4.0), which permits unrestricted use, distribution, and reproduction in any medium, provided the original author and source are credited. 


\section{Introduction}

Weeds can be defined as plants (not necessarily alien) that grow on sites where they are not wanted and which have a detectable economic and/or environmental impact (Richardson et al. 2000, Pyšek et al. 2004). They overlap partly with invasive plants, which are introduced species that cause problems, either in agriculture or in natural areas. According to Pyšek et al. (2004), there have been terminological misunderstandings in studies dealing with plant invasions, partly caused by different perceptions of plant invasions by particular biological disciplines and viewpoints. As shown by these authors, plants encroaching on habitats in which they were not present before can be assessed from the ecological point of view (and termed colonizers), from the biogeographical point of view (invaders, or alien plants in a more general sense), or from the anthropocentric point of view (termed weeds, harmful species, problem plants, noxious plants, pests, etc.).

Paraguay has a total area of $406,752 \mathrm{~km}^{2}$ and is divided into two regions by the Río Paraguay: the Oriental Region and the Occidental Region. The economy is based mainly on agriculture and livestock for export; between the years 2013-2014, approximately $52,381 \mathrm{~km}^{2}$ were dedicated to temporary and permanent crops (DGEEC 2015), particularly soybean $\left(35,000 \mathrm{~km}^{2}\right)$, corn $\left(8,000 \mathrm{~km}^{2}\right)$ and wheat $\left(5,600 \mathrm{~km}^{2}\right)$. During the second half of the twentieth century, global soy production grew tenfold from 27 million tons to 269 million tons (WWF 2016). This increased production was the result of a major expansion in mechanized crops, including other crops that form part of this complex such as wheat, corn, sorghum, canola, among others. It is expected that production will double by 2050 (Bruinsma 2009, WWF 2014). Indeed, Paraguay plays a strong role in this soybean industry; during the 2012-2013 harvest, Paraguay ranked sixth as a global producer and fourth in exports. Itapúa and Alto Paraná have been the highest producing departments, while expansion currently occurs in the departments of San Pedro, Canindeyú and Alto Paraguay (WWF 2016).

Agriculture in Paraguay is carried out on a variety of very distinct soil types in both natural regions. The soils in the Oriental Region are for the most part reddish in colour, resulting from geological events during the Precambrian era and Jurassic and Cretaceous periods (Bertoni and Gorham 1973). These soils include the leptosols and arenosols, which are generally poor in nutrients, and the loamy sand and sandy loam regosols, which are rich in nutrients and where most of the mechanized crops in the region have been carried out (González Erico 2007). In the Occidental Region, most of the soil types are brackish, with a variable content of clay, including planosols, solonetz, gleysols and regosols, where mechanized crops are carried out (Proyecto Sistema Ambiental del Chaco 1992-1997).

Despite being mainly an agricultural country, Paraguay does not have much in terms of research on crop weeds, even in cases where these have a high economic impact on production. Only two sources can actually be considered as references on the subject: Lurvey (1983), the only weed guide available for the country, and the Flora del Paraguay collection (Spichiger and Bocquet 1983-1985, Spichiger 1987-1989, 
Spichiger and Ramella 1990-2002, Ramella and Perret 2008-2014) that superficially covers the behavior of some species, in terms of their weediness, within the families included in the collection. Even herbarium data has proved to be of little use to determine the level of weediness of a species, due to the well-known biases in plant collection towards natural areas.

Weeds have a particular set of skills for survival through mechanisms such as high competitive ability, high seed production, seed production for as long as growing conditions permit, rapid growth throughout vegetative phase to flowering, germination requirements fulfilled in many environments, adaptations for short and long dispersal and great longevity of seeds, self-compatible but not completely autogamous or apomictic, and when cross-pollinated, either pollinated by unspecialized visitors or by wind (Baker 1965, Baker 1974, Lorenzi 2000). Their development in productive areas tends to reduce the quality and quantity of the crop yield, making it difficult to harvest, and in some extreme cases even making it unviable (Guglieri-Caporal et al. 2011). According to Pott et al. (2006), success in controlling invasive plants begins with the floristic inventory of the infesting species and knowledge of the biology of those species that are predominant.

Because of their numerous interactions with humans, their biology, ecology, evolution and community dynamics, weeds are an important subject of study (Kuester et al. 2014). In fact, the study of plant species considered to be weeds in agro-ecosystems is a subject of high interest in the area of invasion ecology, not only because of their natural ability to become established in natural and semi-natural environments (Figueroa et al. 2004, Pyšek et al. 2004) with the consequent great ecological harm they can cause to ecosystems, but also because of the special attributes that make these plant species more invasive (Rejmánek and Richardson 1996), and the way they respond to human disturbance (Hill et al. 2002). Other studies have also focused on revealing taxonomic patterns among natural area invaders and agricultural weeds (Daehler 1998, Kuester et al. 2014), while Conservation Agriculture builds upon weed ecology to generate integrated and sustainable weed management strategies (Bajwa 2014).

Regarding the increasing importance of weed science in a country such as Paraguay, which depends heavily on agricultural production, this work aims to update and enhance the knowledgebase of the most common weeds affecting productive fields throughout the different ecoregions of the country, as well as the generation of relevant information to facilitate their identification and management.

\section{Methods}

Paraguay is a land-locked country located between $19^{\circ}$ and $28^{\circ}$ south latitude and $54^{\circ}$ and $63^{\circ}$ west longitude at the heart of the South American continent, and lies entirely within the Río de la Plata drainage system, second only in size to the Amazon basin. The country is divided into two regions by the Río Paraguay: the Oriental Region, or eastern region, also known as the Paraná region, and the Occidental Region, or western 


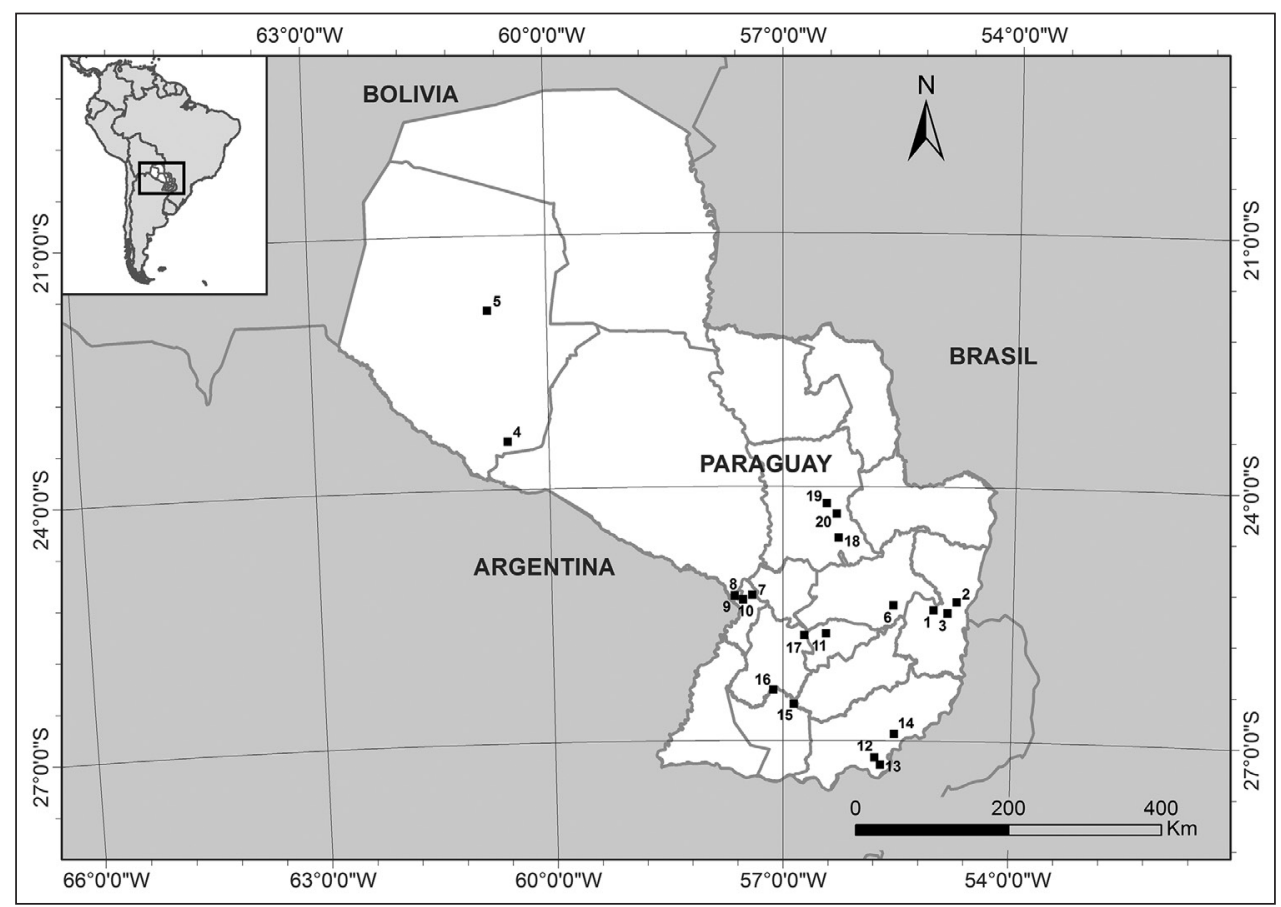

Figure I. Location of the crop weed collection sites

region, also known as the Chaco (part of the Gran Chaco Americano shared by Argentina, Bolivia and Paraguay). Paraguay is divided into 17 departments, 14 of which are located to the east of the Río Paraguay (De Egea et al. 2012).

While building the checklist, we included: 1) a survey of all plant species occurring in key sites, mainly in areas of mechanized soybean, sorghum, sugarcane, corn and rice, where weed management and control was being periodically implemented (Figure 1, Table 1). Each site was surveyed once, at a time when crop fields presented a high level of weed infestation and prior to any weed control activities. Sampling was carried out between 1-3 days, depending on the size of the available crop area per site, by surveying a series of random points (up to 8 per day) to collect the weeds occurring within a 50 meter radius. The data collection included the random collection of plant specimens, georeferencing of the records, the identification of specimens and the resolution of taxonomic problems. Where possible, specimens were collected in quadruplicate; the set of originals were stored in FCQ and duplicates were sent to BM, G and CTES; and 2) the systematization of data available in the literature. Three sources were considered as reference: Lurvey (1983), the Flora del Paraguay collection, and the database of The International Survey of Herbicide Resistant Weeds (Heap 2016), containing a list of the more resistant weed species in the region. Online databases and herbarium resources such as TROPICOS (2016) and the Catálogo de las plantas vasculares del Cono Sur (Zuloaga and Belgrano 2015) were also consulted. 
Table I. Crop weed collection sites.

\begin{tabular}{l|l|l|l}
\hline $\begin{array}{l}\text { Map } \\
\text { key }\end{array}$ & Department & Locality & Type of habitat/crop \\
\hline $\mathbf{1}$ & Alto Paraná & $\begin{array}{l}\text { Colonia Yguazú, Centro de Investigación Agropecuaria del } \\
\text { Paraguay }\end{array}$ & Soybean crops \\
\hline $\mathbf{2}$ & Alto Paraná & Hernandarias & Soybean crops \\
\hline $\mathbf{3}$ & Alto Paraná & $\begin{array}{l}\text { Minga Guazú, Universidad Nacional del Este (UNE) } \\
\text { experimental plots }\end{array}$ & Soybean crops \\
\hline $\mathbf{4}$ & Boquerón & Estancia Toro Mocho & $\begin{array}{l}\text { Cattle pastures and } \\
\text { forest edges }\end{array}$ \\
\hline $\mathbf{5}$ & Boquerón & Mariscal Estigarribia, Estancia Jeroviá & $\begin{array}{l}\text { Soybean and sorghum } \\
\text { crops }\end{array}$ \\
\hline $\mathbf{6}$ & Caaguazú & Juan Eulogio Estigarribia & Soybean crops \\
\hline $\mathbf{7}$ & Central & Areguá & Strawberry crops \\
\hline $\mathbf{8}$ & Central & Asunción & $\begin{array}{l}\text { Vegetable patch/home } \\
\text { garden }\end{array}$ \\
\hline $\mathbf{9}$ & Central & Asunción & Disturbed soils \\
\hline $\mathbf{1 0}$ & Central & $\begin{array}{l}\text { San Lorenzo, Universidad Nacional de Asunción, experimental } \\
\text { plot }\end{array}$ & $\begin{array}{l}\text { Potato, tomato and } \\
\text { cabbage crops }\end{array}$ \\
\hline $\mathbf{1 1}$ & Guairá & Villarrica & Disturbed soils \\
\hline $\mathbf{1 2}$ & Itapúa & Capitán Miranda & Soybean crops \\
\hline $\mathbf{1 3}$ & Itapúa & Nueva Alborada District & Soybean crops \\
\hline $\mathbf{1 4}$ & Itapúa & Pirapó & Soybean crops \\
\hline $\mathbf{1 5}$ & Misiones & San Miguel, Establecimiento La Graciela & Rice crops \\
\hline $\mathbf{1 6}$ & Misiones & Villa Florida & Disturbed soils \\
\hline $\mathbf{1 7}$ & Paraguarí & Villarrica - Paraguarí road & Sugarcane crops \\
\hline $\mathbf{1 8}$ & San Pedro & Barrio San Pedro, Dekalpar crops & Corn crops \\
\hline $\mathbf{1 9}$ & San Pedro & Cruce Liberación & Disturbed soils \\
\hline $\mathbf{2 0}$ & San Pedro & $\begin{array}{l}\text { Instituto Paraguayo de Tecnología Agrícola (IPTA) } \\
\text { experimental plots }\end{array}$ & Fruit crops \\
\hline & & & \\
\hline
\end{tabular}

Data was compiled in a MS Access database. Flowering plant family circumscription follows APG III (Stevens 2001 onwards) and pteridophyte classification follows Smith et al. (2006). In the checklist, families are sorted alphabetically and genera are sorted alphabetically within families. For each taxon we present the accepted name, place and date of publication, synonyms, habit, general distribution in Paraguay, residence status (all species are native except where indicated), and one voucher specimen. Relevant bibliographic citacions are included for taxa recorded in literature, but not found during field surveys nor in the consulted herbaria (BM, CTES, FCQ, and PY). Synonymy follows Zuloaga and Belgrano (2015), except for those taxa covered in more recent literature. Herbarium acronyms used in the text follow Index Herbariorum (Thiers, continuously updated). New reports of taxa for Paraguay are marked with an arrowhead ( ) and those taxa not cited in Zuloaga and Belgrano (2015) for Paraguay but recorded in other publications or databases are marked with an asterisk $\left(^{*}\right)$. 


\section{Data resources}

All occurrence data underpinning the checklist have been uploaded to the Natural History Museum Data Portal (http://dx.doi.org/10.5519/0060042) and are provided as a data supplement to this paper.

\section{Results and discussion}

The list includes a total of 256 taxa (189 species, 10 subspecies, 54 varieties and 3 formas), making up 38 families and 141 genera, of which all except one are angiosperms. There is only one pteridophyte listed, Pteridium arachnoideum (Kaulf.) Maxon, a very close relative of Pteridium aquilinum (L.) Kuhn, a cosmopolitan species with a vigorous vegetative reproductive system (Baker 1974), which is a very common weed worldwide.

The most species-rich families are Poaceae, Asteraceae and Malvaceae, followed by Amaranthaceae, Fabaceae, Solanaceae, Polygonaceae and Cyperaceae (see Table 2). According to Baker (1974), Malvaceae, Amaranthaceae, Cyperaceae and Poaceae are the families which have a higher number of weed species in warm regions, in line with our results.

Over eighty-three percent (213) of the species in the Checklist are herbs, 14.8\% (38) woody species (small trees, shrubs or subshrubs) and only $1.95 \%$ (5) are vines (Figure 2).

The majority of the listed taxa are native to Paraguay (209), representing $81.64 \%$ of the total, including one endemic species (Turnera grandidentata (Urb.) Arbo), while the remaining 47 taxa are introduced. Families with the highest number of non-native species are Poaceae with 16 taxa followed by Brassicaceae with 4 taxa. Most introduced species remain within the status of established plants, except for some species which also show invasive behaviour. Such is the case of Calotropis procera (Aiton) W.T.Aiton, reported for the first time in Paraguay in 1994 (Mereles and Degen 1994), found

Table 2. Number of genera and taxa in the largest families.

\begin{tabular}{l|c|c}
\hline \multicolumn{1}{c|}{ Families } & Genera & Taxa \\
\hline Poaceae & 22 & 50 \\
\hline Asteraceae & 29 & 41 \\
\hline Malvaceae & 11 & 20 \\
\hline Amaranthaceae & 4 & 13 \\
\hline Fabaceae & 9 & 12 \\
\hline Solanaceae & 3 & 12 \\
\hline Polygonaceae & 2 & 11 \\
\hline Cyperaceae & 4 & 10 \\
\hline Euphorbiaceae & 5 & 10 \\
\hline Other families & 52 & 77 \\
\hline
\end{tabular}




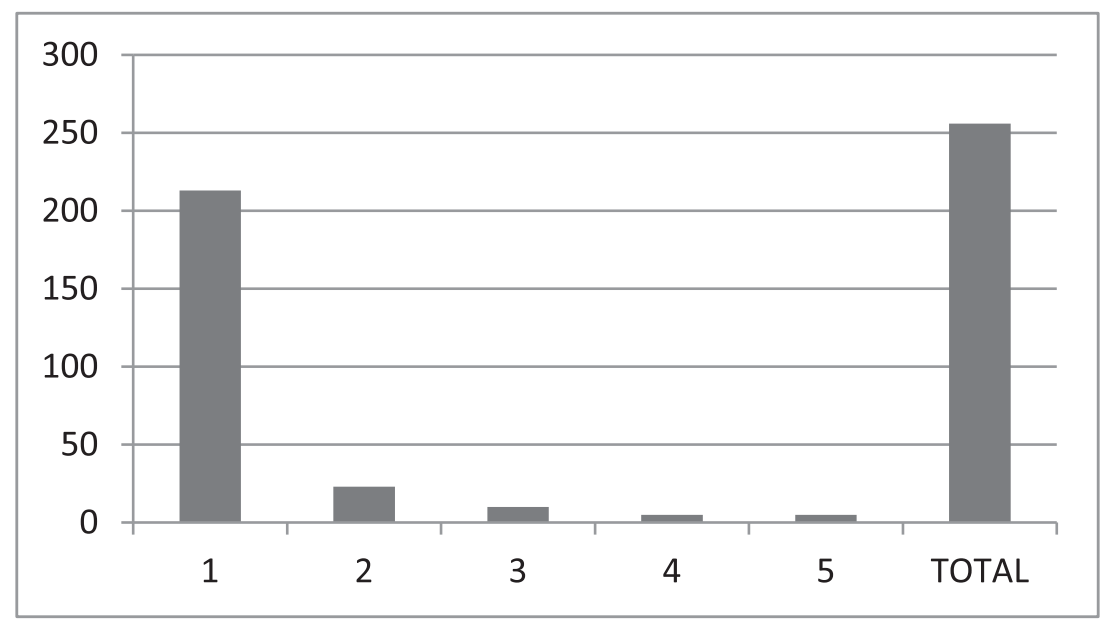

Figure 2. Life forms of the taxa included. I herbs $\mathbf{2}$ subshrubs $\mathbf{3}$ shrubs $\mathbf{4}$ small trees $\mathbf{5}$ vines.

in cattle pastures in northern Paraguay, and currently quite frequent as a weed of difficult eradication within the dry Chaco. Urochloa brizantha (Hochst. ex A.Rich.) R.D.Webster and $U$. decumbens (Stapf) R.D.Webster are also two species that were introduced for grazing (Zuloaga et al. 2014), and are now found in both natural and agro-ecosystems. These three species were also identified as invasive species in Brazil (Base de dados nacional de espécies exóticas invasoras I3N Brasil 2016).

The list includes three new records for the country, collected during crop surveys (Avena sativa (J.Presl.) Barkworth subsp. sativa, Cyperus eragrostis Lam. var. eragrostis and Leonurus cardiaca L. It also includes another seven taxa not listed in Zuloaga \& Belgrano (2015), as being present in Paraguay, but recorded in TROPICOS (2016) or in recent publications (De Egea et al. 2016): Amaranthus hybridus L. subsp. cruentus (L.) Thell., Avena sativa L. subsp. sativa, Capsella bursa-pastoris (L.) Medik., Commelina benghalensis L., Cyperus eragrostis Lam. var. eragrostis, Glandularia cabrerae (Moldenke) Botta, Leonurus cardiaca L., Oldenlandia lancifolia (Schumach.) DC., Tragus australianus and Tridax procumbens L.

Eight taxa mentioned in literature, but not found during field surveys nor in the consulted herbaria, were also included in the checklist: Capsella bursa-pastoris (L.) Medik., Lepidium virginicum L., Raphanus raphanistrum L. (Lurvey 1983), Digitaria aequiglumis (Hack. \& Arechav.) Parodi var. aequiglumis, Echinochloa oryzoides (Ard.) Fritsch (Zuloaga et al. 1994), Paspalum urvillei Steud., Setaria scandens Schrad. (Zuloaga et al. 2014), and Polygonum aviculare L. (Cialdella and Brandbyge 2001).

Nine species were found to be the most frequent and abundant within all crops surveyed: Amaranthus hybridus L., Bidens pilosa L., Conyza bonariensis (L.) Cronquist, Commelina erecta L., Eleusine indica (L.) Gaertn., Euphorbia heterophylla L., Digitaria insularis (L.) Fedde, Richardia brasiliensis Gomes, and Solanum americanum Mill. These species were found in both natural regions of Paraguay and on different soil types, showing a greater plasticity/resilience than the other species found. Overall, they 
are species with high adaptability, fast growth, resistance to drought and high biomass and seed production. It is also likely that the seeds of these weed species accompany the seeds of the harvested grain crops. All these have been listed and highlighted in regional literature on weeds as being problematic species (Ferrari 2012, Guglieri-Caporal 2011, Lorenzi 2000), and with the exception of Commelina erecta and Richardia brasiliensis, are considered among the weeds with greatest herbicide resistance according to The International Survey of Herbicide Resistant Weeds (Heap 2016).

\section{Checklist}

\section{FERNS}

\section{DENNSTAEDTIACEAE}

Pteridium arachnoideum (Kaulf.) Maxon, J. Wash. Acad. Sci. 14: 89. 1924.

Syn.: Pteridium aquilinum (L.) Kuhn var. arachnoideum (Kaulf.) Brade, Pteridium aquilinum ssp. psittacinum (C.Presl) C.Chr, Pteridium aquilinum var. umbrosum H.Christ, nom. illeg., Pteridium caudatum (L.) Maxon ssp. arachnoideum (Kaulf.) Lellinger, Pteridium esculentum (G.Forst.) Cockayne ssp. arachnoideum (Kaulf.) J.A.Thomson, Pteridium psittacinum (C.Presl) Maxon, Pteris aquilina L. var. arachnoidea (Kaulf.) D.C.Eaton, Pteris aquilina var. psittacina (C.Presl) Baker, Pteris arachnoidea Kaulf., Pteris esculenta G.Forst., Pteris psittacina C.Presl.

Herb.

Departmental distribution: Amambay, Caazapá, Canindeyú, Cordillera, Guairá, Paraguarí, San Pedro.

Voucher: J. De Egea et al. 1340 (BM, FCQ, G).

\section{SEED PLANTS}

ACANTHACEAE

Justicia squarrosa Griseb., Abh. Königl. Ges. Wiss. Göttingen 19: 226. 1874.

Syn.: Beloperone ciliata (Nees) Hook.f., B. squarrosa (Griseb.) Lindau, Dianthera ciliata (Nees) Benth. \& Hook.f., hom. illeg., Jacobinia ciliata Nees.

Herb or subshrub.

Departmental distribution: Alto Paraguay, Boquerón, Presidente Hayes.

Voucher: F. Mereles \& S. Soria 9978 (FCQ). 


\section{ALISMATACEAE}

Sagittaria montevidensis Cham. \& Schltdl. subsp. montevidensis, Linnaea 2: 156. 1827.

Syn.: Sagittaria andina Phil., S. montevidensis f. normalis Hauman, nom. inval., S. multinervia Larrañaga, S. pugioniformis L. var. montevidensis (Cham. \& Schltdl.) Kuntze

Herb.

Departmental distribution: Alto Paraguay, Amambay, Boquerón, Caaguazú, Caazapá, Central, Concepción, Cordillera, Guairá, Itapúa, Misiones, Neembucú, Paraguarí, Presidente Hayes, San Pedro.

Voucher: G. Céspedes et al. 445 (BM, FCQ).

\section{AMARANTHACEAE}

Alternanthera ficoidea (L.) P.Beauv., Fl. Oware 2: 66. 1818.

Syn.: Alternanthera polygonoides (L.) R.Br. ex Griseb. var. diffusa (Mart.) Hicken, A. tenella Colla, Bucholzia ficoidea (L.) Mart., Gomphrena ficoidea L.

Herb.

Departmental distribution: Alto Paraguay, Alto Paraná, Amambay, Central, Presidente Hayes, San Pedro.

Voucher: J. De Egea et al. 1230 (FCQ).

Alternanthera paronichyoides A.St.-Hil. subsp. chacoënsis (Morong ex Morong \& Britton) Pedersen, Darwiniana 14: 440. 1967.

Syn.: Alternanthera chacoënsis Morong ex Morong \& Britton, A. ficoidea (L.) Sm. ssp. chacoënsis (Morong ex Morong \& Britton) Pedersen, A. morongii Uline, A. paronychioides var. chacöensis (Morong ex Morong \& Britton) Pedersen, A. paronychioides var. robusta Chodat.

Herb.

Departmental distribution: Alto Paraguay, Boquerón, Caaguazú, Central, Concepción, Cordillera, Guairá, Neembucú, Paraguarí, Presidente Hayes.

Voucher: F. Mereles et al. 10060 (FCQ). 
Alternanthera philoxeroides (Mart.) Griseb. f. philoxeroides, Abh. Königl. Ges. Wiss. Göttingen 24: 36. 1879.

Syn.: Achyranthes paludosa Bunbury, Achyranthes philoxeroides (Mart.) Standl., Alternanthera philoxeroides (Mart.) Griseb.var. acutifolia (Mart.) Hicken, Alternanthera philoxeroides var. obtusifolia (Moq.) Hicken, Bucholzia philoxeroides Mart., B. philoxeroides var. obtusifolia Mart., Mogiphanes philorexoides D.Parodi, Telanthera philoxeroides (Mart.) Moq., T. philoxeroides var. denticulata Seub., T. philoxeroides var. obtusifolia Moq.

Herb aquatic.

Departmental distribution: Alto Paraguay, Central, Presidente Hayes.

Voucher: N. Soria 332 (FCQ).

*Amaranthus hybridus L. subsp. cruentus (L.) Thell., Fl. Adv. Montpellier 205. 1912.

Syn.: Amaranthus cruentus L., A. flavus L., A. paniculatus L.

Herb. Introduced.

Departmental distribution: Alto Paraguay, Guairá, San Pedro.

Voucher: F. Mereles \& S. Soria 10212 (FCQ).

Amaranthus hybridus L. subsp. hybridus, Sp. Pl. 2: 990. 1753.

Syn.: Amaranthus chlorostachys Willd., A. hybridus L. var. hypochondriacus (L.) Rob., A. hybridus var. quitensis (Kunth) Covas, A. hypochondriacus L. var. hypochondriacus, A. quitensis Kunth, A. quitensis var. stuckertianus Thell.

Herb. Introduced.

Departmental distribution: Alto Paraguay, Boquerón, Central, Guairá, Itapúa, Ñeembucú, Presidente Hayes, San Pedro.

Voucher: F. Mereles \& S. Soria 9946 (FCQ).

Amaranthus muricatus (Moq.) Hieron., Pl. Diaph. Fl. Argent. 227. 1882.

Syn.: Euxolus muricatus Moq.

Herb.

Departmental distribution: Alto Paraguay, Boquerón, Presidente Hayes, San Pedro.

Voucher: J. De Egea et al. 1243 (BM, FCQ). 
Amaranthus standleyanus Parodi ex Covas, Darwiniana 5: 339. 1941.

Syn.: Amaranthus parodii Standl., hom. illeg., A. vulgatissimus sensu Thell. non Speg.

Herb.

Departmental distribution: Alto Paraguay, Boquerón, Misiones, Presidente Hayes.

Voucher: F. Mereles et al. 10174 (BM, CTES, FCQ, G).

Amaranthus viridis L., Sp. Pl., ed. 2. 2: 1405. 1763.

Syn.: Amaranthus gracilis Desf., Chenopodium caudatum Jacq., Euxolus caudatus (Jacq.)

Moq., E. viridis (L.) Moq.

Herb.

Departmental distribution: Alto Paraguay, Boquerón, Central, Concepción, Guairá, Paraguarí, Presidente Hayes, San Pedro.

Voucher: F. Mereles \& S. Soria 10186 (FCQ).

Dysphania ambrosioides (L.) Mosyakin \& Clemants, Ukrayins'k. Bot. Zhurn. 59(4): 382. 2002.

Syn.: Ambrina ambrosioides (L.) Spach, A. anthelmintica Spach, A. parvula Phil., Chenopodium ambrosioides L., Ch. ambrosioides var. dentata Fenzl, Ch. ambrosioides f. dentatum (Fenzl) Aellen, Ch.ambrosioides ssp. euambrosioides Aellen, Ch. ambrosioides var. genuinum Willk., Ch. ambrosioides f. genuinum (Willk.) Aellen, Ch. ambrosioides var. integrifolium Fenzl, Ch. ambrosioides f. integrifolium (Fenzl) Aellen, Ch. ambrosioides var. pinnatifidum Willk., Ch. ambrosioides $\mathrm{f}$. pinnatifidum (Willk.) Aellen, Ch. ambrosioides f. rotundantum Aellen, Ch. ambrosioides var. suffruticosum (Willd.) Asch. \& Graebn., Ch. ambrosioides var. typica Speg., Ch. ambrosioides var. typicum (Speg.) Aellen, Ch. suffruticosum Willd., Ch. suffruticosum ssp. remotum Vorosh., Teloxys ambrosioides (L.) W.A.Weber, A. incisa Phil.

Herb.

Departmental distribution: Alto Paraguay, Amambay, Caaguazú, Caazapá, Central, Concepción, Cordillera, Guairá, Itapúa, Paraguarí, Presidente Hayes.

Voucher: R. Degen 120 (FCQ). 
Gomphrena celosioides Mart. f. aureiflora (Chodat) Pedersen, Darwiniana 20: 272. 1976.

Syn.: Gomphrena celosioides var. aureiflora Stuchlik, G. celosioides f. grandifolia Stuchlik, G. celosioides f. parvifolia Stuchlik, G. decumbens Jacq. f. aureiflora Chodat, G. decumbens var. aureiflora (Chodat) Stuchlik, G. hygrophila Mart. f. luteiflora Herzog.

Herb.

Departmental distribution: Guairá, Neembucú, Paraguarí, Presidente Hayes.

Voucher: J. De Egea et al. 1316 (BM, FCQ).

Gomphrena celosioides Mart. var. celosioides, Nova Acta Phys.-Med. Acad. Caes. Leop.-Carol. Nat. Cur. 13: 301. 1826.

Syn.: Gomphrena decumbens Jacq. f. albiflora Chodat \& Hassl., G. decumbens var. albiflora (Chodat \& Hassl.) Stuchlik, G. decumbens var. genuina Stuchlik, nom. inval., G. perennis L. f. ramosissima Stuchlik, Xeraea celosioides (Mart.) Kuntze

Herb.

Departmental distribution: Alto Paraguay, Alto Paraná, Boquerón, Caaguazú, Caazapá, Central, Cordillera, Guairá, Itapúa, Neembucú, Presidente Hayes, San Pedro. Voucher: F. Mereles et al. 9913 (FCQ).

Gomphrena elegans Mart. var. elegans, Nov. Gen. Sp. Pl. 2(1): 17, t. 119. 1826.

Syn.: Gomphrena elegans Mart. var. genuina Stuchlik, nom. inval., G. elegans f. nigrovirida Stuchlik, G. hilariana Moq., G. viridifolia Suess., Xeraea elegans (Mart.) Kuntze, X. hilariana (Moq.) Kuntze

Herb.

Departmental distribution: Canindeyú, Cordillera, Guairá, Paraguarí.

Voucher: E. Zardini \& R. Velázquez 11556 (FCQ).

Gomphrena perennis L. var. perennis, Sp. Pl. 1: 224. 1753.

Syn.: Gomphrena perennis L. ssp. genuina Stuchlik, nom. inval., G. perennisf. parvifolia, G. perennisf. villosa, G. villosa Mart., Xeraea perennis (L.) Kuntze, X. villosa (Mart.) Kuntze

Herb.

Departmental distribution: Alto Paraguay, Boquerón.

Voucher: F. Mereles \& S. Soria 9940 (BM, CTES, FCQ, G). 


\section{APIACEAE}

Cyclospermum leptophyllum (Pers.) Sprague var. leptophyllum, J. Bot. 61: 131. 1923.

Syn.: Apium ammi Urb., A. ammi f. filamentosum (Kuntze) H.Wolff, A. ammi var. filamentosum Kuntze, A. ammi Urb. var. genuinum $\mathrm{H}$.Wolff, A. ammi var. leptophyllum (Pers.) Kuntze, A. ammi f. nanum Kuntze, A. ammi f. pedunculata Chodat. A. laciniatum (DC.) Urb. f. elatius (Hook. \& Arn.) H.Wolff, A. leptophyllum (Pers.) F.Muell. ex Benth., A. ranunculifolium auct. non Kunth, A. ranunculifolium (DC.) Reiche, comb. illeg., Helosciadium laciniatum DC. var. elatius Hook. \& Arn., $H$. leptophyllum (Pers.) DC., H. ranunculifolium DC., Pimpinella leptophylla Pers.

Herb.

Departmental distribution: Alto Paraguay, Alto Paraná, Boquerón, Caazapá, Central, Guairá, Neembucú, Presidente Hayes, San Pedro.

Voucher: J. De Egea et al. 1321 (FCQ).

\section{APOCYNACEAE}

Asclepias curassavica L., Sp. Pl. 1: 215. 1753.

Herb. Introduced.

Departmental distribution: Amambay, Canindeyú, Central, Cordillera, Guairá, Itapúa, Misiones, Paraguarí, Presidente Hayes.

Voucher: F. Mereles et al. 10011 (BM, FCQ, G).

Asclepias mellodora A.St.-Hil., Pl. Rem. Bres. 227. 1824.

Syn.: Asclepias campestris Decne., A. campestris var. angustifolia Kuntze, A. campestris var. schlechteri Kuntze, A. curupi E.Fourn., A. jangadensis S.Moore, A. marginata Decne. var. bodenbenderi Kuntze, $A$. mellodora var. bodenbenderi (Kuntze) Bollwinkel, $A$. mellodora var. minor A.St.-Hil., A. mellodora var. multinervis (E.Fourn.) Bollwinkel, A. multinervis E.Fourn., A. nervosa Decne., A. papillosa Silveira, A. umbellata Vell.

Herb.

Departmental distribution: Amambay, Boquerón, Caazapá, Canindeyú, Central, Concepción, Cordillera, Guairá, Itapúa, Misiones, Neembucú, Paraguarí, Presidente Hayes, San Pedro.

Voucher: J. De Egea et al. 1329 (FCQ). 
Calotropis procera (Aiton) W.T.Aiton, Hort. Kew., ed. 2 2: 78. 1811.

Syn.: Asclepias procera Aiton

Shrub or small tree. Introduced.

Departmental distribution: Alto Paraguay, Boquerón.

Voucher: F. Mereles \& S. Soria 9983 (FCQ, G).

\section{ARALIACEAE}

Hydrocotyle bowlesioides Mathias \& Constance, Bull. Torrey Bot. Club 69: 151. 1942.

Herb.

Departmental distribution: Alto Paraná, Amambay, Caazapá, Central, Guairá, Paraguarí. Voucher: F. Mereles et al. 10098 (FCQ).

\section{ASTERACEAE}

Acanthospermum hispidum DC., Prodr. 5: 522. 1836.

Syn.: Acanthospermum humile DC. var. hispidum (DC.) Kuntze

Herb.

Departmental distribution: Alto Paraguay, Boquerón, Central, Concepción, Cordillera, Neembucú, Paraguarí, Presidente Hayes.

Voucher: A. Quarti P050 (FCQ).

Ageratum conyzoides L., Sp. Pl. 2: 839. 1753.

Syn.: Ageratum conyzoides L. var. hirtum (Lam.) DC., A hirsutum Poir., A. hirtum Lam., A. latifolium Cav., Carelia conyzoides (L.) Kuntze

Herb.

Departmental distribution: Amambay, Caazapá, Canindeyú, Central, Cordillera, Guairá, Itapúa, San Pedro.

Voucher: J. De Egea et al. 1276 (FCQ). 
Ambrosia elatior L., Sp. Pl. 2: 987. 1753.

Syn.: Ambrosia artemisiifolia L., A. artemisiifolia var. elatior (L.) Descourt., A. chilensis Hook. \& Arn., A. peruviana Cabrera, hom. illeg.

Herb.

Departmental distribution: Alto Paraná, Amambay, Boquerón, Caazapá, Central, Concepción, Cordillera, Guairá, Presidente Hayes, San Pedro.

Voucher: F. Mereles et al. 10090 (FCQ, G).

Ambrosia tenuifolia Spreng., Syst. Veg. (ed. 16) 3: 851. 1826.

Herb.

Departmental distribution: Central, Paraguarí, Presidente Hayes.

Voucher: F. Mereles 5263 (CTES, FCQ).

Aspilia montevidensis (Spreng.) Kuntze var. montevidensis, Revis. Gen. Pl. 3[3]: 129. 1898.

Syn.: Aspilia arillata (DC.) Griseb., A. buphthalmiflora (DC.) Griseb., A. calendulacea (DC.) Griseb., A. montevidensis (Spreng.) Kuntze var. setosa (Griseb.) Cabrera, $A$. setosa Griseb., Leighia arillata DC., L. buphthalmiflora DC., L. calendulacea DC., Verbesina montevidensis Spreng., Wedelia montevidensis (Spreng.) B.L.Turner

Herb.

Departmental distribution: Amambay, Caaguazú, Caazapá, Canindeyú, Central, Concepción, Cordillera, Guairá, Itapúa, Misiones, Paraguarí, Presidente Hayes, San Pedro. Voucher: J. De Egea et al. 1334 (BM, CTES, FCQ, G).

Aspilia silphioides Benth. \& Hook. f., Gen. Pl. 2(1): 372. 1873.

Syn.: Gymnopsis helianthoides DC., Leighia silphioides Hook. \& Arn., Wedelia silphioides (Hook. \& Arn.) B.L.Turner

Herb.

Departmental distribution: Amambay, Boquerón, Central, Concepción, Cordillera, Neembucú, Presidente Hayes.

Voucher: F. Mereles \& S. Soria 9953 (BM, FCQ, G). 
Baccharis salicifolia (Ruiz \& Pav.) Pers., Syn. Pl. 2: 425.1807.

Syn.: Baccharis alamanii DC., B. araucana Phil., B. pallida Heering ex Reiche, B. chilquilla DC., nom. superfl., B. coerulescens DC., B. confertifolia Colla var. confertifolia, B. cuervii Phil., B. fevillei DC., B. glutinosa Pers. var. incisa Heering, B. huydobriana J.Rémy, B. iresinoides Kunth, B. kraussei Heering ex Reiche, B. lanceolata Kunth emend. Heering, B. linifolia DC., hom. illeg., B. longifolia DC., B. longipes Kunze ex DC., B. marginalis DC., B. marginalis var. araucana (Phil.) Heering, B. marginalis var. linifolia (Phil.) Heering, B. marginalis var. longipes (Kunze ex DC.) Heering, B. parviflora (Ruiz \& Pav.) Pers., B. purpurascens Heering, P. salicifolia (Ruiz \& Pav.) F.H.Hellwig, B. salicifolia (Ruiz \& Pav.) Pers. var. longifolia (DC.) Cuatrec., B. sphaerocephala Hook. \& Arn. var. krausei (Heering) Malag., comb. illeg., B. viscosa (Ruiz \& Pav.) Kuntze var. nigricans Kuntze, Molina parviflora Ruiz \& Pav., M. salicifolia Ruiz \& Pav., M. striata Ruiz \& Pav., Pingraea marginalis (DC.) F.H.Hellwig

Shrub.

Departmental distribution: Alto Paraguay, Boquerón, Central, Itapúa, Presidente Hayes.

Voucher: F. Mereles et al. 9917 (BM, FCQ, G).

Bidens pilosa L. var. pilosa, Sp. Pl. 2: 832. 1753.

Syn.: Bidens californica DC., B. hispida Kunth, B. hirsuta Nutt., hom. illeg., B. leucantha Willd. f. discoidea Sch.Bip., B. leucantha var. pilosa (L.) Griseb., B. leucantha var. sundaica (Blume) Hassk., B. montaubanii Phil., B. pilosa L. var. brevifoliata Hieron, B. pilosa var. discoidea Sch.Bip., B. pilosa var. dubia (Cass.) O.E.Schulz, B. pilosa var. minor (Blume) Sherff, B. reflexa Link, B. sundaica Blume, B. sundaica var. minor Blume, Kerneria dubia Cass.

Herb.

Departmental distribution: Alto Paraguay, Alto Paraná, Amambay, Caaguazú, Canindeyú, Central, Cordillera, Guairá, Itapúa, Misiones, Paraguarí, Presidente Hayes, San Pedro. Voucher: J. De Egea et al. 1236 (FCQ).

Bidens subalternans DC. var. subalternans, Prodr. 5: 600. 1836.

Syn.: Bidens quadrangularis DC., B. platensis Manganaro

Herb.

Departmental distribution: Alto Paraguay, Amambay, Caaguazú, Caazapá, Central, Cordillera, Guairá, Paraguarí.

Voucher: N. Soria 5736 (FCQ, MO). 
Chrysolaena cognata (Less.) Dematteis, Bol. Soc. Argent. Bot. 44(1-2): 157. 2009.

Syn.: Cacalia cognata (Less.) Kuntze, Vernonia cinerascens Sch.Bip. ex Baker, nom. nud., $V$. cognata Less., $V$. senecionea Mart. ex DC., $V$. senecionea var. adenocarpa DC., $V$. senecionea f. calvata Chodat

Herb.

Departmental distribution: Alto Paraná, Amambay, Caaguazú, Canindeyú, Central, Concepción, Cordillera, Guairá, Itapúa, Misiones, Paraguarí, San Pedro.

Voucher: M. Dematteis et al. 3138 (CTES, FCQ).

Chrysolaena platensis (Spreng.) H.Rob., Proc. Biol. Soc. Washington 101: 957. 1988.

Syn.: Cacalia platensis (Spreng.) Kuntze, Conyza platensis Spreng., Vernonia platensis (Spreng.) Less., V. virens Sch.Bip. ex Baker f. robustior Chodat

Subshrub.

Departmental distribution: Amambay, Concepción, Cordillera, Guairá, Misiones, Paraguarí, San Pedro.

Voucher: J. De Egea et al. 1335 (BM, CTES, FCQ, G).

Conyza bonariensis (L.) Cronquist var. angustifolia (Cabrera) Cabrera, Man. Fl. Alrededores Buenos Aires 481. 1953.

Syn.: Conyza linearis DC., Erigeron bonariensis L. var. angustifolius Cabrera

Herb.

Departmental distribution: Central, Misiones.

Voucher: F. Mereles et al. 10176 (FCQ).

Conyza bonariensis (L.) Cronquist var. bonariensis, Bull. Torrey Bot. Club 70: 632. 1943.

Syn.: Conyza hispida Kunth, C. plebeja Phil., Erigeron bonariensis L., E. bonariensis f. grisea Chodat \& Hassl., Marsea bonariensis (L.) V.M.Badillo

Herb.

Departmental distribution: Alto Paraguay, Amambay, Caaguazú, Caazapá, Central, Cordillera, Guairá, Paraguarí, Presidente Hayes.

Voucher: F. Mereles 6450 (FCQ). 
Conyza sumatrensis (Retz.) E.Walker var. leiotheca (S.F.Blake) Pruski \& G.Sancho, Novon 16(1): 98-99, f. 1. 2006.

Syn.: Conyza bilbaoana J.Remy, C. bonariensis (L.) Cronquist var. leiotheca (S.F.Blake) Cuatrec., C. elata Kunth \& Bouché, C. floribunda Kunth, C. floribunda var. laciniata Cabrera, C. myriocephala J.Remy, C. sumatrensis (Retz.) E. Walker var. floribunda (Kunth) J.B.Marshall, Erigeron bilbaoanus (J.Remy) Cabrera, E. bonariensis L. var. floribundum (Kunth) Cuatrec., E. bonariensis f. glabrata Speg., E. bonariensis var. leiothecus S.F.Blake, E. floribundus (Kunth) Sch.Bip., Marsea bonariensis (L.) V.M.Badillo var. leiotheca (S.F.Blake) V.M.Badillo

Herb.

Departmental distribution: Alto Paraná, Amambay, Caaguazú, Central, Guairá, Itapúa, Paraguarí.

Voucher: N. Soria 2684 (FCQ, MO).

Eclipta prostrata (L.) L., Mant. Pl. Altera 286. 1771.

Syn.: Eclipta alba (L.) Hassk., E. erecta L., nom. illeg., Galinsoga oblongifolia (Hook.) DC., Polygyne inconspicua Phil., Verbesina alba L., V. conyzoides Trew, V. prostrata L., Wiborgia oblongifolia Hook.

Herb.

Departmental distribution: Alto Paraguay, Alto Paraná, Amambay, Boquerón, Canindeyú, Central, Concepción, Cordillera, Guairá, Misiones, Presidente Hayes, San Pedro.

Voucher: F. Mereles \& J. De Egea 10129 (FCQ).

Emilia fosbergii Nicolson, Phytologia 32(1): 34. 1975.

Syn.: Emilia javanica auct. non (Burm.) C.B.Rob., E. sagittata auct. non (Vahl) DC.

Herb. Introduced.

Departmental distribution: Alto Paraná, Amambay, Caaguazú, Canindeyú, Central, Cordillera, Guairá, Misiones, San Pedro.

Voucher: F. Mereles \& J. De Egea 10147 (FCQ). 
Erechtites valerianifolius (Link ex Spreng.) DC. f. valerianifolius, Prodr. 6: 295. 1838.

Syn.: Crassocephalum valerianifolium (Link ex Spreng.) Less., Erechtites gardneriana Cabrera, E. valerianifolius (Wolf) DC., nom. inval., Gynura rosea Ridl., Senecio valerianifolius Wolf ex Link, nom. nud., Senecio valerianifolius Desf., hom. illeg., Senecio valerianifolius Gardner, hom. illeg., Senecio valerianifolius Link ex Spreng., Sonchus erythropappus Meyen \& Walp. ex Walp.

Herb.

Departmental distribution: Alto Paraná, Amambay, Caaguazú, Caazapá, Canindeyú, Central, Cordillera, Guairá, Itapúa, Paraguarí, San Pedro.

Voucher: J. De Egea et al. 1237 (FCQ).

Eupatorium inulifolium Kunth, Nov. Gen. Sp. 4(15): 85 (ed. fol.). 1818.

Shrub.

Departmental distribution: Alto Paraná, Amambay, Boquerón, Caaguazú, Caazapá, Canindeyú, Central, Concepción, Cordillera, Guairá, Itapúa, Paraguarí, Presidente Hayes, San Pedro.

Voucher: F. Mereles 9930 (BM, FCQ, G).

Flaveria bidentis (L.) Kuntze, Revis. Gen. Pl. 3(3): 148. 1898.

Syn.: Ethulia bidentis L., Eupatorium chilense Molina, Flaveria bidentis (L.) B.L.Rob., comb. superf., F. bonariensis DC., F. capitata Juss. ex Sm., F. chilensis (Molina) J.F.Gmel., F. contrayerba (Cav.) Pers., Milleria chiloensis Juss., nom. nud., M. contrayerba Cav., Vermifuga corymbosa Ruiz \& Pav., nom. illeg.

Herb.

Departmental distribution: Alto Paraguay, Boquerón, Concepción, Presidente Hayes. Voucher: F. Mereles 5667 (CTES, FCQ).

Galinsoga parviflora Cav., Icon. 3(2): 41-42, pl. 281. 1796.

Syn.: Adventina parviflora Raf., Galinsoga quinqueradiata Ruiz \& Pav., nom. superfl., Wiborgia acmella Roth, W. parviflora (Cav.) Kunth

Herb.

Departmental distribution: Amambay, Central, Guairá.

Voucher: G. Céspedes 422 (FCQ). 
Gamochaeta americana (Mill.) Wedd., Chlor. And. 1: 151. 1856.

Syn.: Gamochaeta americana var. alpina Wedd., Gamochaeta guatemalensis (Gand.) Cabrera, Gnaphalium americanum Mill., Gnaphalium consanguineum Gaudich., Gnaphalium guatemalense Gand., Gnaphalium purpureum L. var. americaum (Mill.) Klatt.

Herb.

Departmental distribution: Alto Paraná, Amambay, Central, Cordillera, Presidente Hayes. Voucher: G. Céspedes 432 (FCQ).

Gamochaeta calviceps (Fernald) Cabrera, Bol. Soc. Argent. Bot. 9: 368. 1961.

Syn.: Gnaphalium calviceps Fernald

Herb.

Departmental distribution: Alto Paraguay, Amambay, Caaguazú, Central, Concepción, Cordillera, Paraguarí, San Pedro.

Voucher: J. De Egea et al. 1227 (BM, FCQ).

Gamochaeta coarctata (Willd.) Kerguélen, Lejeunia 120: 104. 1987.

Syn.: Gamochaeta spicata Cabrera, Gnaphalium coarctatum Willd., Gnaphalium purpureum L. var. spicatum Baker, nom. illeg., Gnaphalium spicatum Lam., nom. illeg.

Herb.

Departmental distribution: Alto Paraguay, Alto Paraná, Caazapá, Central, Cordillera, Guairá, Itapúa, San Pedro.

Voucher: J. De Egea et al. 1322 (FCQ).

Gamochaeta pensylvanica (Willd.) Cabrera, Bol. Soc. Argent. Bot. 9: 375. 1961.

Syn.: Gamochaeta platensis (Cabrera) Cabrera, Gnaphalium pensylvanicum Willd., Gnaphalium peregrinum Fernald, Gnaphalium platense Cabrera, Gnaphalium purpureum L. ssp. pensylvanica (Willd.) O. Bolòs \& Vigo, Gnaphalium purpureum var. spathulatum Baker, Gnaphalium spathulatum Lam., hom. illeg.

Herb.

Departmental distribution: Alto Paraguay, Alto Paraná, Boquerón, Canindeyú, Central, Cordillera, Guairá, Paraguarí, Presidente Hayes.

Voucher: I. Basualdo 395 (FCQ). 
Lepidaploa remotiflora (Rich.) H.Rob., Proc. Biol. Soc. Washington 103: 491.1990.

Syn.: Vernonia hirtiflora Sch.Bip. ex Baker, V. lithospermoides Baker, V. remotiflora Rich., V. remotiflora var. tricholepis (DC.) Baker, V. sessiliflora Willd. ex Less., $V$. tricholepis DC.

Herb.

Departmental distribution: Alto Paraguay, Alto Paraná, Amambay, Caaguazú, Caazapá, Canindeyú, Central, Concepción, Cordillera, Guairá, Itapúa, Misiones, Ñeembucú, Paraguarí, Presidente Hayes, San Pedro.

Voucher: J. De Egea et al. 1223 (BM, FCQ).

Parthenium hysterophorus L., Sp. Pl. 2: 988. 1753.

Syn.: Argyrochaeta bipinnatifida Cav., Echetrosis pentasperma Phil., Parthenium glomeratum Rollins, P. lobatum Buckley, P. pinnatifidum Stokes, nom. superfl., Villanova bipinnatifida Ortega

Herb.

Departmental distribution: Alto Paraguay, Amambay, Presidente Hayes.

Voucher: J. De Egea et al. 142 (BM, CTES, FCQ, G, MO).

Pluchea sagittalis (Lam.) Cabrera, Bol. Soc. Argent. Bot. 3: 36. 1949.

Syn.: Conyza sagittalis Lam., Epaltes brasiliensis DC., Gnaphalium suaveolens Vell., Pluchea quitoc DC., P. suaveolens (Vell.) Kuntze

Herb.

Departmental distribution: Alto Paraguay, Amambay, Boquerón, Caaguazú, Caazapá, Central, Concepción, Cordillera, Guairá, Paraguarí, Presidente Hayes.

Voucher: F. Mereles et al. 10048 (FCQ, G).

Praxelis clematidea (Griseb.) R.M.King \& H.Rob., Phytologia 20: 194. 1970.

Syn.: Eupatorium catarium Veldkamp, E. clematideum Griseb., hom. illeg., E. pauciflorum auct. non Kunth, E. urticifolium auct. non L., E. urticifolium L. f. var. clematideum Hieron. ex Kuntze, E. urticifolium f. var. nanum Hieron. ex Kuntze

Herb. 
Departmental distribution: Alto Paraguay, Amambay, Boquerón, Caaguazú, Canindeyú, Central, Concepción, Cordillera, Guairá, Misiones, Neembucú, Paraguarí, Presidente Hayes, San Pedro.

Voucher: W.J. Hahn 1613 (MO, PY).

Senecio brasiliensis (Spreng.) Less var. brasiliensis, Linnaea 6: 249. 1831.

Syn.: Cineraria brasiliensis Spreng.

Herb.

Departmental distribution: Alto Paraná, Amambay, Caaguazú, Caazapá, Canindeyú, Itapúa, Misiones.

Voucher: N. Soria 2513 (CTES, MO).

Senecio grisebachii Baker var. grisebachii, Fl. Bras. 6(3): 313. 1884.

Herb.

Departmental distribution: Alto Paraguay, Amambay, Caaguazú, Caazapá, Canindeyú, Central, Cordillera, Guairá, Itapúa, Misiones, Neembucú, Paraguarí, Presidente Hayes, San Pedro.

Voucher: F. Mereles et al. 10005 (FCQ).

Senecio hieronymi Griseb., Abh. Königl. Ges. Wiss. Göttingen 24: 205. 1879.

Syn.: Senecio charaguensis Cuatrec., S. tafiensis Cabrera

Subshrub.

Departmental distribution: Alto Paraná, Canindeyú, Central, Guairá, Paraguarí. Voucher: J. De Egea et al. 1331 (BM, FCQ).

Solidago chilensis Meyen var. chilensis, Reise Erde 1: 311. 1834.

Syn.: Aster sagei Phil., Solidago coquimbana Phil., S. floribunda Phil., S. laxiflora Phil., S. linearifolia DC., S. linearifolia var. brachypoda Speg., S. microglossa DC. var. linearifolia (DC.) Baker, S. parviflora Phil., S. recta Phil., S. valdiviana Phil.

Herb.

Departmental distribution: Alto Paraguay, Boquerón, Cordillera.

Voucher: I. Basualdo 111 (FCQ). 
Solidago microglossa DC., Prodr. 5: 332. 1836.

Syn.: Solidago chilensis Meyen var. megapotamica (DC.) Cabrera, S. microglossa DC. var. megapotamica DC.

Herb.

Departmental distribution: Alto Paraguay, Alto Paraná, Amambay, Caaguazú, Caazapá, Central, Concepción, Cordillera, Guairá, Paraguarí, Presidente Hayes, San Pedro. Voucher: M. Ortiz 1004 (FCQ).

Soliva sessilis Ruiz \& Pav., Syst. Veg. Fl. Peruv. Chil. 1: 215. 1798.

Syn.: Gymnostyles alata Spreng., G. barclayana (DC.) Steud., G. chilensis Spreng., G. pterosperma Juss., Soliva alata (Spreng.) DC., S. barclayana DC., S. daucifolia Nutt., S. microloma Phil., S. neglecta Cabrera, S. pterosperma (Juss.) Less., S. sessilis Ruiz \& Pav. var. barclayana (DC.) Baker, S. valdiviana Phil.

Herb.

Departmental distribution: Alto Paraná, Concepción, Cordillera, Guairá, Misiones, Paraguarí, San Pedro.

Voucher: L. Pérez 448 (FCQ).

Sonchus oleraceus L., Sp. Pl. 2: 794. 1753.

Syn.: Sonchus gracilis Phil., S. rivularis Phil.

Herb. Introduced.

Departmental distribution: Alto Paraguay, Alto Paraná, Boquerón, Caazapá, Canindeyú, Central, Guairá, San Pedro.

Voucher: J. De Egea et al. 1238 (BM, FCQ).

Synedrellopsis grisebachii Hieron. \& Kuntze, Revis. Gen. Pl. 3(3): 180. 1898.

Syn.: Synedrella nodiflora Griseb. ex Kuntze, nom. nud.

Herb.

Departmental distribution: Alto Paraguay, Amambay, Boquerón, Central, Itapúa, San Pedro.

Voucher: J. De Egea \& F. Mereles 1357 (BM, CTES, FCQ, G). 
Tagetes minuta L., Sp. Pl. 2: 887. 1753.

Syn.: Tagetes bonariensis Pers., T. glandulifera Schrank, T. glandulosa Link, T. porophyllum Vell.

Herb.

Departmental distribution: Alto Paraguay, Amambay, Central, Cordillera, Paraguarí, Presidente Hayes.

Voucher: A. Schinini 2401 (FCQ).

Taraxacum officinale F.H.Wigg., Prim. Fl. Holsat. 56. 1780.

Syn.: Leontodon taraxacum L., L. vulgaris Lam., nom. illeg., Taraxacum dens-leonis Desf., T. subspathulatum A.J.Richards, T. vulgare (Lam.) Schrank., nom. illeg.

Herb. Introduced.

Departmental distribution: Alto Paraná, Caazapá, Guairá.

Voucher: R. Degen 982 (FCQ).

*Tridax procumbens L., Sp. Pl. 2: 900. 1753.

Syn.: Amellus pedunculatus Ortega ex Willd., nom. inval., Balbisia canescens Pers., $B$. divaricata Cass., B. elongata Willd., B. pedunculata Ortega ex Hoffmans., nom. illeg., Tridax procumbens L. var. canescens (Pers.) DC., T. procumbens var. ovatifolia B.L.Rob. ex Greenm.

Herb.

Departmental distribution: Boquerón, Central, Concepción, San Pedro.

Voucher: F. Mereles \& S. Soria 9944 (BM, FCQ, G).

Vernonanthura tweedieana (Baker) H.Rob., Phytologia 73: 74. 1992.

Syn.: Chrysocoma arborea Vell., Vernonia tweedieana Baker

Shrub.

Departmental distribution: Alto Paraguay, Alto Paraná, Amambay, Caaguazú, Central, Cordillera, Guairá, Itapúa, Misiones, Paraguarí, Presidente Hayes, San Pedro.

Voucher: F. Mereles et al. 9907 (FCQ, G). 
Vernonia incana Less., Linnaea 4: 277. 1829.

Syn.: Cacalia incana (Less.) Kuntze, Vernonia inmunis Griseb.

Herb.

Departmental distribution: Alto Paraná, Amambay, Central, Neembucú, Paraguarí, Presidente Hayes.

Voucher: M. Ortiz 551 (FCQ).

\section{BORAGINACEAE}

Heliotropium leiocarpum Morong, Ann. New York Acad. Sci. 7: 168. 1893.

Syn.: Heliotropium leiocarpum Morong f. albiflora Chodat, H. leiocarpum f. minor Chodat

Subshrub.

Departmental distribution: Alto Paraná, Central, Cordillera, Paraguarí, Presidente Hayes.

Voucher: A. Krapovickas \& C.L. Cristóbal 44544 (FCQ).

\section{BRASSICACEAE}

Brassica rapa L., Sp. Pl. 2: 666. 1753.

Syn.: Brassica campestris L.

Herb. Introduced.

Departmental distribution: Alto Paraguay, Boquerón, Central.

Voucher: M. Ortíz 283 (FCQ).

*Capsella bursa-pastoris (L.) Medik., Pfl.-Gatt. 85. 1792.

Syn.: Thlaspi bursa-pastoris L.

Herb. Introduced.

Departmental distribution: Itapúa.

Voucher: E. Lurvey 181 (Lurvey 1983). 
Lepidium didymum L., Syst. Nat., ed. 12. 2: 433. 1767.

Syn.: Coronopus didymus (L.) Sm., C. didymus var. macrocarpus Muschl., C. didymus var. procumbens Muschl., C. leptocarpus Boelcke, C. leptocarpus var. microcarpus Boelcke, Lepicochlea americana Rojas Acosta, Senebiera didyma (L.) Pers., S. pinnatifica DC.

Herb.

Departmental distribution: Alto Paraná, Amambay, Caazapá, Central, Cordillera.

Voucher: I. Basualdo 5223 (FCQ).

Lepidium virginicum L., Sp. Pl. 2: 645. 1753.

Herb. Introduced.

Departmental distribution: Amambay, Caaguazú, Central, Guairá, Itapúa.

Voucher: E. Lurvey 359 (Lurvey 1983).

Raphanus raphanistrum L., Sp. Pl. 2: 669. 1753.

Herb. Introduced.

Departmental distribution: Amambay, Cordillera, Itapúa.

Voucher: E. Lurvey 374 (Lurvey 1983).

Raphanus sativus L., Sp. Pl. 2: 669. 1753.

Herb. Introduced.

Departmental distribution: Amambay, Central, Cordillera, Misiones, San Pedro. Voucher: J. De Egea et al. 1268 (FCQ).

\section{CAMPANULACEAE}

Lobelia xalapensis Kunth, Nov. Gen. Sp. 3: 315. 1819.

Syn.: Dortmannia cliffortiana Kuntze var. xalapensis (Kunth) Kuntze, D. mollis (Graham) Kuntze, D. monticola (Kunth) Kuntze, D. ocimoides (Kunze) Kuntze, Lobelia cliffortiana L., L. cliffortiana var. xalapensis (Kunth) A.Gray, L. mollis Graham, L. monticola Kunth, L. ocimoides Kunze, L. palmaris Willd. ex Roem. \& Schult., Rapuntium monticulum (Kunth) C.Presl, $R$. xalapense (Kunth) C.Presl 
Herb.

Departmental distribution: Alto Paraguay, Amambay, Boquerón, Caazapá, Canindeyú, Central, Concepción, Cordillera, Guairá, Paraguarí, San Pedro.

Voucher: J. De Egea et al. 1317 (BM, FCQ).

Triodanis perfoliata (L.) Nieuwl. subsp. biflora (Ruiz \& Pav.) Lammers, Novon 16(1): 72.2006.

Syn.: Campanula biflora Ruiz \& Pav., C. ludoviciana Torr. ex A.Gray, nom. nud., C. montevidensis Spreng., Dysmicodon californicum Nutt., D. ovatum Nutt., Legouzia biflora (Ruiz \& Pav.) Britton, Pentagonia biflora (Ruiz \& Pav.) Kuntze, Specularia biflora (Ruiz \& Pav.) Fisch. \& A.Gray, S. californica (Nutt.) Vatke, S. ovata (Nutt.) Vatke, S. perfoliata (L.) A.DC., S. perfoliata f. ramosa Arechav., S. perfoliata f. rigida Arechav., Triodanis biflora (Ruiz \& Pav.) Greene, T. perfoliata (L.) Nieuwl. var. biflora (Ruiz \& Pav.) T.R.Bradley

Herb.

Departmental distribution: Alto Paraná, Central, Guairá, Paraguarí.

Voucher: J. De Egea et al. 1313 (FCQ).

Wablenbergia linarioides (Lam.) A.DC., Monogr. Campan. 158. 1830.

Syn.: Campanula arida Kunth, C. chilensis Molina, C. linarioides Lam., Wahlenbergia arida (Kunth) Griseb., W. linarioides (Lam.) A.DC. var. arida (Kunth) A.DC., W. linarioides var. micrantha Phil.

Herb.

Departmental distribution: Alto Paraná, Amambay, Caaguazú, Caazapá, Canindeyú, Central, Guairá, Itapúa, Neembucú, Paraguarí.

Voucher: F. Mereles et al. 10088 (FCQ, G).

\section{CARYOPHYLLACEAE}

Cerastium rivulariastrum Möschl \& Pedersen, Darwiniana 16(1-2): 118. 1970.

Herb.

Departmental distribution: Canindeyú, Central, Guairá, Misiones, Paraguarí.

Voucher: G. Céspedes 435 (FCQ). 
Drymaria cordata (L.) Willd. ex Roem. \& Schult., Syst. Veg. (ed. 15 bis) 5: 406. 1819.

Syn.: Drymaria cordata (L.) Willd. ex Roem. \& Schult. var. pacifica Mizush., Holosteum cordatum $\mathrm{L}$.

Herb.

Departmental distribution: Central, Cordillera, Guairá, Paraguarí.

Voucher: E. Zardini \& R. Velázquez 27329 (FCQ, MO).

Paronychia communis Cambess. var. communis, Fl. Bras. Merid. (quarto ed.) 2(15): 186. 1829.

Herb.

Departmental distribution: Alto Paraná, Canindeyú, Cordillera, Paraguarí.

Voucher: F. Mereles et al. 10073 (FCQ, G).

Stellaria media (L.) Cirillo var. gymnocalyx Trautv., Acta Horti Petrop. 1: 33. 1881.

Syn.: Stellaria glabra Raunk.

Herb. Introduced.

Departmental distribution: Itapúa.

Voucher: E. Lurvey 205 (CTES).

\section{CLEOMACEAE}

Cleome aculeata L. var. aculeata, Syst. Nat., ed. 12. 3: 232. 1768.

Herb.

Departmental distribution: Alto Paraguay, Central, Concepción, Cordillera, Guairá, Itapúa, Paraguarí, Presidente Hayes.

Voucher: M. Ortíz 317 (FCQ).

Cleome tucumanensis Iltis, Brittonia 12: 284. 1960.

Syn.: Cleome flexuosa Griseb., nom. illeg.

Herb.

Departmental distribution: Alto Paraguay, Boquerón, Central, Presidente Hayes.

Voucher: F. Mereles \& S. Soria 9941 (FCQ, G). 


\section{COMMELINACEAE}

${ }^{*}$ Commelina benghalensis L., Sp. Pl. 1: 41.1753.

Herb. Introduced.

Departmental distribution: Cordillera, Guairá, Paraguarí, San Pedro.

Voucher: J. De Egea et al. 1273 (FCQ).

Commelina diffusa Burm.f. var. diffusa, Fl. Indica 18, pl. 7, f. 2.1768.

Syn.: Commelina cayennensis Rich., C. cayennensis var. pubescens Griseb., C. longicaulis Jacq., C. nudiflora auct. non L.

Herb.

Departmental distribution: Concepción, Guairá, Misiones, Neembucú, Paraguarí. Voucher: F. Mereles \& J. De Egea 10149 (FCQ, G).

Commelina diffusa Burm.f. var. gigas (Small) Faden, Ann. Missouri Bot. Gard. 80: 213. 1993.

Syn.: Commelina gigas Small

Herb.

Departmental distribution: Boquerón, Central, Cordillera, Guairá, Itapúa, Paraguarí. Voucher: F. Mereles et al. 9919 (FCQ, G).

Commelina erecta L. var. angustifolia (Michx.) Fernald, Rhodora 42: 439. 1940.

Syn.: Commelina angustifolia Michx., C. virginica L. var. angustifolia (Michx.) C.B.Clarke

Herb.

Departmental distribution: Boquerón, Cordillera.

Voucher: F. Mereles \& S. Soria 9964 (FCQ, G).

Commelina erecta L. var. erecta, Sp. Pl. 1: 41. 1753.

Syn.: Commelina elegans Kunth, C. pohliana Seub., C. sulcata Willd., C. virginica auct. non $\mathrm{L}$., C. virginica L. var. australis C.B.Clarke 
Herb.

Departmental distribution: Alto Paraguay, Amambay, Canindeyú, Central, Neembucú, Paraguarí, Presidente Hayes, San Pedro.

Voucher: F. Mereles 9936 (BM, FCQ, G).

Commelina platyphylla Klotzsch, Reis. Br.-Guiana 3: 897. 1849.

Syn.: Commelina balansae (C.B.Clarke) Herter, C. platyphylla var. balansae C.B.Clarke Herb.

Departmental distribution: Boquerón, Caaguazú, Central, Concepción, Cordillera, Itapúa, Misiones, Neembucú, Paraguarí, Presidente Hayes.

Voucher: F. Mereles \& J. De Egea 10127 (FCQ).

\section{CONVOLVULACEAE}

Ipomoea cordatotriloba Dennst. var. australis (O'Donell) D.F.Austin, Taxon 37(1): 185. 1988.

Syn.: Ipomoea trichocarpa Elliot, nom. illeg., I. trichocarpa Elliott var. australis O’Donell,

Vine.

Departmental distribution: Amambay, Caaguazú, Central, Cordillera, Guairá, Paraguarí, San Pedro.

Voucher: E. Zardini 9984 (FCQ, MO).

Ipomoea grandifolia (Dammer) O’Donell, Arq. Mus. Paraense 9: 222. 1952.

Syn.: Ipomoea triloba auct. non L., Jacquemontia grandifolia Dammer

Vine.

Departmental distribution: Alto Paraguay, Caazapá, Canindeyú, Central, Concepción, Cordillera, Guairá, Misiones, Neembucú, Paraguarí, San Pedro.

Voucher: F. Mereles \& J. De Egea 10140 (FCQ, G).

Ipomoea nil (L.) Roth, Catal. Bot. 1: 36. 1797.

Syn.: Convolvulus nil L., C. tomentosus Vell., Ipomoea cuspidata Ruiz \& Pav., I. longicuspis Meisn., nom. illeg., I. scabra Forssk., Pharbitis cuspidata (Ruiz \& Pav.) G.Don 
Vine.

Departmental distribution: Boquerón, Caazapá, Canindeyú, Central, Cordillera, Guairá, Itapúa, Paraguarí, Presidente Hayes, San Pedro.

Voucher: J. De Egea et al. 1318 (BM, FCQ, G).

\section{CUCURBITACEAE}

Cucurbitella asperata (Gillies ex Hook. \& Arn.) Walp., Repert. Bot. Syst. 6: 50. 1846.

Syn.: Cucurbita asperata Gillies ex Hook. \& Arn., Cucurbita urkupinana Cárdenas, Cucurbitella cucumifolia (Griseb.) Cogn., Cucurbitella duriaei (Naudin) Cogn., Cucurbitella integrifolia Cogn., Cucurbitella integrifolia Cogn. var. glabrior Cogn, Cucurbitella urkupinana (Cárdenas) C.Jeffrey, Prasopepon cucumifolius Griseb., P. durieui Naudin, Schizostigma asperatum (Gillies ex Hook. \& Arn.) Arn., comb. illeg.

Vine.

Departmental distribution: Alto Paraguay, Boquerón, Central, Concepción, Paraguarí, Presidente Hayes, San Pedro.

Voucher: J. De Egea et al. 1226 (BM, FCQ).

\section{CYPERACEAE}

Bulbostylis capillaris (L.) Kunth ex C.B.Clarke var. capillaris, Fl. Brit. India 6(19): 652. 1893.

Syn.: Abildgaardia capillaris (L.) Lye, Fimbristylis capillaris (L.) A.Gray, Isolepis capillaris (L.) Roem. \& Schult., Scirpus capillaris L., Stenophyllus capillaris (L.) Britton

Herb.

Departmental distribution: Alto Paraguay, Caaguazú, Caazapá, Guairá, Paraguarí. Voucher: J. De Egea et al. 1327 (FCQ).

Cyperus aggregatus Endl., Cat. Horti Vindob. 1: 93. 1842.

Syn.: Cyperus aggregatus (Willd.) Endl. var. gigas (Lindm.) Guagl., C. argentinus Boeck., C. cayennensis (Lam.) Britton, nom. illeg., C. cayennensis var. gigas (Lindm.) Barros, C. cayennensis var. umbellato-flavus (C.B.Clarke) Barros, C. flavomariscus Griseb., C. flavus (Vahl) Nees, nom. illeg., C. flavus var. aggregatus (Willd.) Kük., C. flavus var. angustatus Kük., C. flavus var. argentinus (Boeck.) Kük. ex Osten, C. flavus var. gigas (Lindm.) Kük., C. retrorsus Chapm. var. australis (Lindm.) Kük., 
Didymia cyperomorpha Phil., Kyllinga cayennensis Lam., Mariscus aggregatus Willd., M. cayennensis (Lam.) Urb., M. cylindricus Elliot var. australis Lindm., M. flavus Vahl, M. flavus var. gigas Lindm., C. flavus var. laevis (Kunth) Kük., M. flavus $\mathrm{f}$. umbellato-flava C.B.Clarke

Herb.

Departmental distribution: Alto Paraguay, Alto Paraná, Amambay, Canindeyú, Central, Cordillera, Guairá, Itapúa, Misiones, Neembucú, Paraguarí, Presidente Hayes, San Pedro.

Voucher: J. De Egea et al. 1289 (BM, FCQ).

Cyperus eragrostis Lam. var. eragrostis, Tabl. Encycl. i. 196. 1791.

Syn.: Cyperus compressus Jacq., C. declinatus Moench, C. eragrostis Lam. f. latifrons Kük., C. eragrostis $\mathrm{f}$. tener Kük., C. monandrus Roth, $C$. vegetus Willd., C. vegetus var. obtusangulus Kuntze

Herb.

Departmental distribution: Guairá.

Voucher: F. Mereles \& S. Soria 9949 (FCQ, G).

Cyperus esculentus L. var. leptostachyus Boeck., Linnaea 36: 290. 1870.

Syn.: Chlorocyperus phymatodes (Muhl.) Palla, Cyperus esculentus L. var. phymatodes (Muhl.) Kük., Cyperus phymatodes Muhl.

Herb. Introduced.

Departmental distribution: Alto Paraguay, Alto Paraná, Central, Cordillera, Itapúa. Voucher: J. De Egea et al. 325 (BM, MO).

Cyperus haspan L. var. haspan, Sp. Pl. 1: 45. 1753.

Syn.: Cyperus adenophorus Schrad., C. americanus (Boeck.) Palla, C. autummalis (Rottb.) Vahl, C. cayennensis Willd. ex Link, C. efoliatus Boeck., C. haspan L. var. adenophorus (Schrad.) Kük., C. haspan var. americanus Boeck., Cyperus haspan ssp. juncoides (Lam.) Kük., C. haspan var. riparius (Schrad. ex Nees) Kük., C. juncoides Lam., C. riparius Schrad. ex Nees, Scirpus autumnalis Rottb., hom. illeg.

Herb. 
Departmental distribution: Alto Paraguay, Caaguazú, Caazapá, Canindeyú, Central, Cordillera, Guairá, Itapúa, Misiones, Neembucú, Paraguarí.

Voucher: F. Mereles \& S. Soria 10201 (FCQ).

Cyperus odoratus L., Sp. Pl. 1: 46. 1753.

Syn.: Cyperus acicularis (Nees) Steud., C. cephalophorus J.Presl \& C.Presl, C. conglobatus Link, C. densiflorus G.Mey., C. engelmannii Steud., C. ferax Rich., C. ferax var. acicularis (Nees) Kük., C. ferax var. bulbiferus Barros, C. ferax var. conglobatus (Link) Kük., C. ferax ssp. engelmanni (Steud.) Kük., C. ferax var. maximilianii (Schrad. ex Nees) Boeck., C. ferax ssp. speciosus (Vahl) Kük., C. ferox Vahl, C. flexuosus Vahl, C. hamiltonii Kunth, C. huarmensis (Kunth) M.C.Johnst., C. jubaeflorus Rudge, C. maximilianii (Schrad. ex Nees) Griseb., C. pohlianus (Nees) Kuntze, C. speciosus Vahl, Diclidium lenticulare Schrad. ex Nees, D. lomentaceum Nees, D. maximilianii Schrad. ex Nees, D. odoratum Schrad. ex Nees, D. uliginosum Schrad. ex Nees, Mariscus ferax (Rich.) C.B.Clarke, M. pohlianus Nees, Torulinium confertum Desv. ex Ham., T. ferax (Rich.) Urb., T. odoratum (L.) S.S.Hooper

Herb.

Departmental distribution: Alto Paraguay, Alto Paraná, Amambay, Boquerón, Caaguazú, Caazapá, Central, Concepción, Cordillera, Guairá, Itapúa, Misiones, Neembucú, Paraguarí, Presidente Hayes, San Pedro.

Voucher: F. Mereles \& S. Soria 9965 (FCQ, G).

Cyperus rotundus L., Sp. Pl. 1: 45. 1753.

Syn.: Chlorocyperus rotundus (L.) Palla

Herb.

Departmental distribution: Caazapá, Cordillera, Itapúa.

Voucher: F. Mereles et al. 9920 (FCQ).

Cyperus tenuis Sw., Prodr. 20. 1788.

Syn.: Cyperus caracasanus Kunth

Herb.

Departmental distribution: Caaguazú, Misiones, Paraguarí.

Voucher: F. Mereles \& J. De Egea 10136 (FCQ). 
Eleocharis montana (Kunth) Roem. \& Schult., Syst. Veg. 2: 153. 1817.

Syn.: Eleocharis consanguinea Kunth, Eleocharis montana var. nodulosa (Roth) Svenson, Eleocharis nodulosa (Roth) Schult., Eleocharis nodulosa f. trigyna Barros, Eleogenus nodulosus (Roth) Nees, Scirpus montanus Kunth, S. nodulosus Roth

Herb aquatic.

Departmental distribution: Alto Paraguay, Alto Paraná, Amambay, Caaguazú, Caazapá, Canindeyú, Central, Concepción, Cordillera, Guairá, Itapúa, Misiones, Ñeembucú, Paraguarí, Presidente Hayes, San Pedro.

Voucher: G. Céspedes \& al 443 (BM, FCQ).

Rhynchospora corymbosa (L.) Britton var. corymbosa, Trans. New York Acad. Sci. 11: 84. 1892.

Syn.: Calyptrostylis fascicularis Nees, C. florida (Rudge) Nees, Dichromena corymbosa (L.) J.F.Macbr., Rhynchospora aurea Vahl, R. corymbosa (L.) Britton var. florida (Rudge) Kük., $R$. florida (Rudge) Schult., $R$. surinamensis (Rottb.) Nees, Schoenus floridus Rudge, Schoenus surinamensis Rottb., Scirpus corymbosus L.

Herb.

Departmental distribution: Alto Paraguay, Amambay, Caaguazú, Canindeyú, Central, Concepción, Cordillera, Guairá, Itapúa, Misiones, Neembucú, Paraguarí, Presidente Hayes, San Pedro.

Voucher: G. Céspedes et al. 444 (FCQ).

\section{EUPHORBIACEAE}

Acalypha communis Müll.Arg., Linnaea 34: 23. 1865.

Syn.: Acalypha. agrestis Morong ex Britton, A. apicalis N.E.Br., A. communis Müll.Arg. var. agrestis (Morong ex Britton) Chodat, A. communisf. grandifolia Chodat \& Hassl., A. communis var. guaranitica Chodat \& Hassl., A. communis var. hirta (Spreng.) Müll. Arg., comb. illeg., A. communis var. salicifolia Pax \& K.Hoffm., A. communis var. saltensis Pax \& K.Hoffm., A. communis var. tomentella Müll.Arg., A. communis var. tomentosa Müll.Arg., A. cordobensis Müll.Arg., A. cordobensis var. rotundata Griseb., A. gracilis Griseb., hom. illeg., A. hirta Spreng., hom. illeg., A. montevidensis Klotzsch ex Pax \& K.Hoffm., nom. nud., A. paraguariensis Chodat \& Hassl., A. variabilis Klotzsch ex Baill. var. angustifolia Baill., Ricinocarpus cordobensis (Müll.Arg.) Kuntze. 
Herb or subshrub.

Departmental distribution: Alto Paraguay, Alto Paraná, Amambay, Boquerón, Caazapá, Canindeyú, Central, Concepción, Cordillera, Guairá, Itapúa, Neembucú, Paraguarí, Presidente Hayes, San Pedro.

Voucher: F. Mereles 9929 (BM, CTES, FCQ, G).

Cnidoscolus albomaculatus (Pax) I.M.Johnst., Contr. Gray Herb. 68: 86. 1923.

Syn.: Jatropha albomaculata Pax, J. albomaculata var. nana (Chodat \& Hassl.) Pax, J. albomaculata var. stimulosissima (Chodat \& Hassl.) Pax, J. albomaculata var. subcuneata Pax, J. vitifolia Mill. f. nana Chodat \& Hassl., J. vitifolia f. stimulosissima Chodat \& Hassl.

Herb or shrub.

Departmental distribution: Alto Paraguay, Amambay, Boquerón, Caazapá, Canindeyú, Central, Concepción, Cordillera, Guairá, Itapúa, Neembucú, Paraguarí, Presidente Hayes, San Pedro.

Voucher: F. Mereles \& S. Soria 10181 (FCQ).

Croton glandulosus L., Syst. Nat., ed. 10. 2: 1275. 1759.

Syn.: Croton divaricatus Sw., C. glandulosus L. var. scordioides (Lam.) Müll.Arg., C. scordioides Lam.

Herb.

Departmental distribution: Alto Paraná, Amambay, Boquerón, Caazapá, Canindeyú, Central, Concepción, Cordillera, Guairá, Itapúa, Paraguarí.

Voucher: J. De Egea et al. 1372 (FCQ).

Euphorbia heterophylla L., Sp. Pl. 1: 453. 1753.

Syn.: Poinsettia heterophylla (L.) Klotzsch \& Garcke

Herb.

Departmental distribution: Alto Paraná, Amambay, Caazapá, Canindeyú, Central, Concepción, Cordillera, Guairá, Itapúa, Paraguarí.

Voucher: F. Mereles et al. 9921 (BM, FCQ, G). 
Euphorbia hirta L. var. hirta, Sp. Pl. 1: 454. 1753.

Syn.: Chamaesyce hirta (L.) Millsp., Euphorbia pilulifera auct. non L., E. pilulifera L. var. guaranitica Chodat \& Hassl.

Herb. Introduced.

Departmental distribution: Alto Paraguay, Alto Paraná, Central, Itapúa, Paraguarí.

Voucher: F. Mereles et al. 10096 (FCQ).

Euphorbia hypericifolia L., Sp. Pl. 1: 454. 1753.

Syn.: Chamaesyce hypericifolia (L.) Millsp., Euphorbia boliviana Rusby

Herb.

Departmental distribution: Alto Paraguay, Itapúa.

Voucher: F. Mereles et al. 9923 (FCQ, G).

Euphorbia prostrata Aiton, Hort. Kew. 2: 139. 1789.

Syn.: Chamaesyce prostrata (Aiton) Small

Herb.

Departmental distribution: Central, Guairá.

Voucher: F. Mereles 10123 (FCQ).

Euphorbia selloi (Klotzsch \& Garcke) Boiss., Prodr. 15(2.1): 50. 1862.

Syn.: Anisophyllum selloi Klotzsch \& Garcke, Chamaesyce selloi (Klotzsch \& Garcke) Croizat, Ch. selloi var. brevisemina Croizat, Euphorbia hassleriana Chodat, E. hebegyne Pax \& K.Hoffm. ex Emrich, E. selloi var. setosa auct. non Boiss., E. setosa (Boiss.) Müll.Arg.

Herb.

Departmental distribution: Central, Guairá.

Voucher: F. Mereles 9938 (FCQ). 
Euphorbia serpens Kunth var. serpens, Nov. Gen. Sp. 2: 52.1817.

Syn.: Chamaesyce serpens (Kunth) Small

Herb.

Departmental distribution: Alto Paraguay, Amambay, Boquerón, Central, Presidente Hayes.

Voucher: F. Mereles \& S. Soria 9986 (BM, FCQ, G).

Jatropha hieronymi Kuntze, Revis. Gen. Pl. 3(2): 287. 1898.

Shrub or small tree.

Departmental distribution: Alto Paraguay, Boquerón.

Voucher: F. Mereles 10192 (FCQ).

\section{FABACEAE}

Aeschynomene falcata (Poir.) DC. var. falcata, Prodr. 2: 322. 1825.

Syn.: Aeschynomene apoloana Rusby, A. falcata (Poir.) DC. var. paucijuga Benth., Hedysarum diffusum Vell., H. falcatum Poir.

Herb.

Departmental distribution: Caaguazú, Canindeyú, Central, Concepción, Cordillera, Guairá, Paraguarí.

Voucher: F. Mereles 1883 (FCQ).

Baubinia forficata Link subsp. pruinosa (Vogel) Fortunato \& Wunderlin, Darwiniana 27: 550. 1986.

Syn.: Bauhinia candicans Benth., B. forficata auct. non Link, B. forficata auct. non Hook. \& Arn., B. forficata var. candicans (Benth.) Hassl. ex Latzina, B. forficata Link var. pruinosa (Vogel) Hassl., B. pruinosa Vogel, Pauletia candicans (Benth.) A.Schmitz, P. pruinosa (Vogel) A.Schmitz

Shrub or tree.

Departmental distribution: Alto Paraná, Caaguazú, Central, Cordillera, Itapúa. Voucher: F. Mereles et al. 9905 (FCQ, G). 
Chamaecrista rotundifolia (Pers.) Greene var. rotundifolia, Pittonia 4(20): 31. 1899.

Syn.: Cassia bifoliolata DC. ex Collad., Cassia bifoliolata var. pentandra (Raddi) Desv., Cassia bifoliolata var. rotundifolia (Pers.) Desv., comb. illeg., Cassia fabaginifolia Kunth, Cassia monophylla Vell., Cassia pentandra Raddi, Cassia pentandria Larrañaga, hom. illeg., Cassia rotundifolia Pers., Cassia tenuivenosa A.P.D.Jones., Chamaecrista bifoliolata (DC. ex Collad.) Greene.

Herb or subshrub.

Departmental distribution: Alto Paraguay, Amambay, Caaguazú, Caazapá, Central, Cordillera, Guairá.

Voucher: J. De Egea et al. 1337 (BM, FCQ).

Crotalaria incana L., Sp. Pl. 2: 716. 1753.

Syn.: Chrysocalyx schimperi Hochst. ex A.Rich., Crotalaria affinis DC., Crotalaria criocaula S.Schauer, Crotalaria cubensis DC., Crotalaria diffusa Vell., hom. illeg., Crotalaria eriocaula S.Schauer, Crotalaria glabrescens Andersson, hom. illeg., Crotalaria herbacea Schweig. ex Schrank, Crotalaria hirta Lag., hom. illeg., Crotalaria incana L. f. microphylla Chodat \& Hassl., Crotalaria megapotamica Burkart, Crotalaria montana A.Rich., Crotalaria picensis Phil., Crotalaria pubescens Moench, Crotalaria purpurascens Lam., Crotalaria radiata Merr., Crotalaria setifera DC., Lupinus rotundifolius Sessé \& Moç.

Herb or shrub.

Departmental distribution: Alto Paraguay, Amambay, Boquerón, Caaguazú, Caazapá, Canindeyú, Central, Concepción, Cordillera, Guairá, Itapúa, Paraguarí, Presidente Hayes, San Pedro.

Voucher: J. De Egea et al. 1240 (FCQ).

Desmodium cuneatum Hook. \& Arn., Bot. Misc. 3: 195. 1832.

Syn.: Desmodium brevipes Vogel, Meibomia brevipes (Vogel) Kuntze, M. cuneata (Hook. \& Arn.) Kuntze

Subshrub.

Departmental distribution: Alto Paraguay, Amambay, Caazapá, Canindeyú, Central, Concepción, Cordillera, Guairá, Itapúa, Misiones, Neembucú, Paraguarí, Presidente Hayes, San Pedro.

Voucher: J. De Egea et al. 1338 (BM, FCQ). 
Desmodium tortuosum (Sw.) DC., Prodr. 2: 332. 1825.

Syn.: Desmodium purpureum (Mill.) Fawc. \& Rendle, hom. illeg., Hedysarum purpureum Mill., H. tortuosum Sw., Meibomia purpurea (Mill.) Vail, M. tortuosa (Sw.) Kuntze.

Subshrub.

Departmental distribution: Amambay, Caaguazú, Concepción, Itapúa, Paraguarí. Voucher: F. Mereles \& F. González Parini 7837 (FCQ).

Indigofera bongardiana (Kuntze) Burkart var. bongardiana, Darwiniana 4: 171. 1942.

Syn.: Anila bongardiana Kuntze, Indigofera gracilis Bong. ex Benth.

Herb.

Departmental distribution: Amambay, Caaguazú, Canindeyú, Central, Itapúa, Misiones, San Pedro.

Voucher: F. Mereles \&J. De Egea 10132 (FCQ, G).

Lupinus gibertianus C.P.Sm. var. gibertianus, Spec. Lupinorum 206. 1940.

Syn.: Cytisus heptaphyllus Vell., Lupinus aspersus C.P.Sm., L. bonplandianus C.P.Sm., L. hassleranus C.P.Sm., L. heptaphyllus (Vell.) Hassl., L. heptaphyllus f. hilarianus Benth., L. heptaphyllus f. typicus Hassl., nom. inval., L. hilarianus Benth., nom. superfl., L. propedubius C.P.Sm., L. sanctae-anae C.P.Sm., L. subumbellatus C.P.Sm.

Herb.

Departmental distribution: Itapúa, Neembucú, San Pedro.

Voucher: J. De Egea et al. 1283 (FCQ).

Senna morongii (Britton) H.S.Irwin \& Barneby, Mem. New York Bot. Gard. 35: 364. 1982.

Syn.: Cassia acinacicarapa Rusby, C. cochabambae Herzog, C. morongii Britton, C. rojasiana Hassl., C. tomentosa L. f. var. paucijuga Kuntze

Subshrub.

Departmental distribution: Alto Paraguay, Boquerón, Concepción, Misiones, Paraguarí, Presidente Hayes.

Voucher: F. Mereles \& S. Soria 9958 (BM, FCQ, G). 
Senna obtusifolia (L.) H.S.Irwin \& Barneby, Mem. New York Bot. Gard. 35: 252. 1982.

Syn.: Cassia humilis Collad., C. obtusifolia L., C. tora L. var. humilis Collad., C. toroides Raf., C. toroides Roxb., nom. nud., Diallobus falcatus Raf., nom. illeg., D. uniflorus Raf., Emelista tora (L.) Britton \& Rose, Senna toroides Roxb.

Herb or subshrub.

Departmental distribution: Alto Paraguay, Alto Paraná, Boquerón, Caaguazú, Caazapá, Canindeyú, Central, Cordillera, Guairá, Itapúa, Paraguarí, Presidente Hayes. Voucher: J. De Egea et al. 1312 (FCQ).

Senna occidentalis (L.) Link., Handbuch 2: 140. 1831.

Syn.: Cassia caroliniana Walter, C. ciliata Raf., C. falcata L., C. foetida Pers., nom. illeg., C. macradena Collad., C. obliquifolia Schrank, C. occidentalis L., C. occidentalis var. aristata Collad., C. planisiliqua L., C. planisiliqua Lam., hom. illeg., C. plumieri DC., Ditremexa occidentalis (L.) Britton \& Rose ex Britton \& Wilson, Senna occidentalis (L.) Roxb., comb. superfl.

Herb or subshrub.

Departmental distribution: Alto Paraguay, Amambay, Boquerón, Caaguazú, Caazapá, Canindeyú, Central, Concepción, Cordillera, Guairá, Misiones, Neembucú, Paraguarí, Presidente Hayes, San Pedro.

Voucher: J. De Egea et al. 1262 (FCQ).

Vicia epetiolaris Burkart var. epetiolaris, Darwiniana 14: 182. 1966.

Vine.

Departmental distribution: Central, Misiones, Presidente Hayes. Voucher: G. Céspedes 439 (FCQ).

\section{LAMIACEAE}

Hyptis lappacea Benth., Labiat. Gen. Sp. 103. 1833.

Syn.: Hyptis cinerea Morong, H. cinerea var. genuina Briq., nom. inval., H. cinerea var. stenophylla Briq., H. globifera auct. non G.Mey., H. michelii Briq. ex Micheli, H. trichoneura Briq. ex Micheli, Mesosphaerum cinereum (Morong) Briq., M. lappaceum (Benth.) Kuntze, M. trichoneurum (Briq. ex Micheli) Briq. 
Herb.

Departmental distribution: Alto Paraguay, Alto Paraná, Amambay, Boquerón, Caaguazú, Caazapá, Central, Concepción, Cordillera, Guairá, Neembucú, Paraguarí, Presidente Hayes.

Voucher: R. Degen 1434 (FCQ).

Leonotis nepetifolia (L.) R.Br., Hortus Kew. (2nd ed.) 3: 409. 1811.

Syn.: Phlomis nepetaefolia L.

Herb. Introduced.

Departmental distribution: Amambay, Caazapá, Central, Cordillera, Paraguarí, San Pedro.

Voucher: E. Zardini 8223 (FCQ, MO).

Leonurus cardiaca L., Sp. Pl. 2: 584.1753.

Herb. Introduced.

Departmental distribution: Guairá.

Voucher: R. Degen et al. 3811 (FCQ).

Leonurus japonicus Houtt., Nat. Hist. 2(9): 366, t. 57, f. 1. 1778.

Syn.: Leonurus sibiricus auct. non L.

Herb. Introduced.

Departmental distribution: Alto Paraná, Caazapá, Central, Guairá, Itapúa, Paraguarí. Voucher: R. Degen 1516 (FCQ).

Scutellaria racemosa Pers., Syn. Pl. 2(1): 136. 1806.

Syn.: Scutellaria bonariensis Willd. ex Benth., S. hastata Larrañaga, S. heterophylla Willd. ex Benth., S. rumicifolia Kunth, S. rojasii Briq.

Herb.

Departmental distribution: Alto Paraguay, Caaguazú, Canindeyú, Central, Concepción, Cordillera, Itapúa, Misiones, Ñeembucú, Paraguarí, Presidente Hayes, San Pedro.

Voucher: J. De Egea 1299 (BM, FCQ). 


\section{LYTHRACEAE}

Cuphea carthagenensis (Jacq.) J.F.Macbr., Publ. Field Mus. Nat. Hist., Bot. Ser. 8: 124. 1930.

Syn.: Cuphea balsamona Cham. \& Schltdl., Lythrum carthagenense Jacq.

Herb or subshrub.

Departmental distribution: Amambay, Caazapá, Canindeyú, Central, Concepción, Cordillera, Guairá, Itapúa, Misiones, Neembucú, Paraguarí, Presidente Hayes, San Pedro.

Voucher: N. Soria 3111a (FCQ).

Cuphea racemosa (L.f.) Spreng. subsp. racemosa, Syst. Veg. (ed. 16) 2: 455. 1825.

Syn.: Cuphea obtusifolia Koehne ex Bacig., C. origanifolia Cham. \& Schltdl., C. racemosa var. discolor Lourteig, Lythrum racemosum L.f.

Herb or subshrub.

Departmental distribution: Alto Paraná, Amambay, Caaguazú, Caazapá, Canindeyú, Central, Concepción, Cordillera, Guairá, Itapúa, Misiones, Neembucú, Paraguarí, Presidente Hayes, San Pedro.

Voucher: G. Céspedes 428 (FCQ).

Heimia salicifolia Link., Icon. Pl. Select. 63, t. 28. 1822.

Syn.: Nesaea salicifolia Kunth

Shrub or subshrub.

Departmental distribution: Alto Paraguay, Alto Paraná, Amambay, Boquerón, Caaguazú, Caazapá, Canindeyú, Central, Concepción, Cordillera, Guairá, Itapúa, Misiones, Neembucú, Paraguarí, Presidente Hayes, San Pedro.

Voucher: F. Mereles et al. 10012 (BM, FCQ, G).

Pleurophora saccocarpa Koehne, Bot. Jahrb. Syst. 2(5): 426. 1882.

Syn.: Pleurophora annulosa Koehne, P. saccocarpa Koehne var. fiebrigii Koehne, P. saccocarpa var. glabrescens Koehne, P. saccocarpa var. hirtella Koehne, P. saccocarpa var. velutina Koehne

Shrub. 
Departmental distribution: Alto Paraguay, Alto Paraná, Amambay, Boquerón, Canindeyú, Central, Concepción, Misiones, Neembucú, Paraguarí, Presidente Hayes, San Pedro.

Voucher: F. Mereles \& J. De Egea 10137 (BM, FCQ, G).

\section{MALVACEAE}

Abutilon pauciflorum A.St.-Hil., Fl. Bras. Merid. 1: 206. 1827.

Syn.: Abutilon melanocarpum A.St.-Hil. \& Naudin, A. mollissimum auct. non (Cav.) Sweet, A. pauciflorum A.St.-Hil. var. cano-tomentosum Hassl., A. pauciflorum $\mathrm{f}$. longe-corniculatum Hassl., $A$. pedunculare auct. non Humb., Bonpl. \& Kunth, $A$. rugosulum Hochr. ex Chodat \& Hassl.

Shrub.

Departmental distribution: Central, Concepción, Cordillera, Guairá, Neembucú, Paraguarí, Presidente Hayes.

Voucher: I. Basualdo 17 (FCQ).

Corchorus hirtus L., Sp. Pl., ed. 2. 2: 747. 1762.

Syn.: Corchorus hirtus L. var. cuyabensis K.Schum, C. hirtus var. orinocensis (Kunth) K.Schum., C. orinocensis Kunth, C. pilolobus Link

Herb or subshrub.

Departmental distribution: Alto Paraguay, Alto Paraná, Amambay, Boquerón, Canindeyú, Central, Concepción, Cordillera, Neembucú, Paraguarí, Presidente Hayes, San Pedro.

Voucher: M. Dematteis et al. 2927 (FCQ).

Malvastrum coromandelianum (L.) Gracke subsp. coromandelianum, Bonplandia 5(18): 295.1857.

Syn.: Malva coromandeliana L., Malva tricuspidata Aiton, Malvastrum carpinifolium A.Gray, Malvastrum coromandelianum (L.) Garcke f. breviaristata Hassl., Malvastrum tricuspidatum (Aiton) A.Gray, Malveopsis coromandeliana (L.) Morong

Subshrub.

Departmental distribution: Alto Paraguay, Amambay, Boquerón, Caaguazú, Central, Concepción, Cordillera, Guairá, Itapúa, Paraguarí, Presidente Hayes, San Pedro. Voucher: F. Mereles et al. 9926 (FCQ). 
Melochia canescens Cristóbal, Bonplandia 9(1-2): 43. 1996.

Subshrub.

Departmental distribution: Alto Paraguay, Boquerón, Neembucú, Presidente Hayes. Voucher: F. Mereles \& S. Soria 9947 (BM, CTES, FCQ, G).

Melochia hermannioides A.St.-Hil., Fl. Bras. Merid. 1(4): 163. 1825.

Syn.: Melochia hermannioides f. heterophylla Hassl., M. hermannioides var. lacinulata (K.Schum. \& Hassl.) Hassl., M. hermannioides var. lanceolata Hassl., M. hermannioides f. typica Hassl., nom. inval., M. lacinulata K.Schum. \& Hassl., Melochia parvifolia Kunth f. roseiflora K.Schum. \& Hassl.

Herb or subshrub.

Departmental distribution: Caaguazú, Canindeyú, Central, Concepción, Cordillera, Guairá, Misiones, Neembucú, Paraguarí, Presidente Hayes, San Pedro.

Voucher: F. Mereles \& J. De Egea 10141 (FCQ, G).

Melochia ministella Cristóbal, Bonplandia 9(1-2): 46. 1996.

Subshrub.

Departmental distribution: Alto Paraguay, Concepción, Itapúa, Presidente Hayes. Voucher: F. Mereles et al. 9925 (BM, FCQ, G).

Melochia pyramidata L. var. hieronymi K.Schum., Fl. Bras. 12(3): 35. 1886.

Syn.: M. pyramidata L. f. intermedia Hassl., M. pyramidata var. pseudotomentosa Hassl., M. pyramidata f. transitoria K.Schum. \& Hassl., Melochia tomentosa L. var. mattogrossensis R.E.Fr.

Subshrub.

Departmental distribution: Alto Paraguay, Amambay, Caaguazú, Caazapá, Canindeyú, Central, Concepción, Cordillera, Guairá, Itapúa, Misiones, Neembucú, Paraguarí, Presidente Hayes, San Pedro.

Voucher: C.L. Cristóbal \& A. Krapovickas 2293 (FCQ). 
Melochia pyramidata L. var. pyramidata, Sp. Pl. 2: 674. 1753.

Subshrub.

Departmental distribution: Alto Paraguay, Amambay, Boquerón, Central, Concepción, Itapúa, Misiones, Paraguarí, Presidente Hayes, San Pedro.

Voucher: R. Spichiger et al. RS2621 (FCQ).

Pavonia sidifolia Kunth, Nov. Gen. Sp. 5: 283. 1822.

Syn.: Asterochlaena sidifolia (Kunth) Hassl., A. sidifolia ssp. diuretica (A.St.-Hil.) Hassl., A. sidifolia var. paraguayensis Hassl., Pavonia diuretica A.St.-Hil.

Shrub or subshrub.

Departmental distribution: Alto Paraguay, Amambay, Canindeyú, Central, Concepción, Cordillera, Guairá, Misiones, Paraguarí, Presidente Hayes, San Pedro.

Voucher: A. Schinini 23872 (FCQ).

Pseudabutilon callimorphum (Hochr.) R.E.Fr. var. callimorphum, Kungl. Svenska Vetenskapsakad. Handl. 43(4): 105. 1908.

Syn.: Sida callimorpha Hochr., Wissadula callimorpha (Hochr.) Hassl.

Subshrub.

Departmental distribution: Alto Paraguay, Boquerón, Concepción, Presidente Hayes. Voucher: F. Mereles \& S. Soria 9939 (BM, CTES, FCQ, G).

Sida cordifolia L., Sp. Pl. 2: 684. 1753.

Syn.: Malvastrum cordifolium Rojas Acosta, Sida altheifolia Sw., S. cordifolia L. var. breviaristata Monteiro, S. rotundifolia Lam.

Subshrub.

Departmental distribution: Alto Paraguay, Amambay, Boquerón, Caaguazú, Caazapá, Central, Concepción, Cordillera, Guairá, Paraguarí, Presidente Hayes, San Pedro. Voucher: J. De Egea et al. 1264 (FCQ). 
Sida linifolia Cav., Diss. 1, Diss. Bot. 14 (t. 2, f. 1). 1785.

Syn.: Sida fiebrigii Ulbr., S. linifolia Juss. ex Cav. f. flaviflora Chodat \& Hassl.

Herb.

Departmental distribution: Amambay, Caaguazú, Central, Concepción, Cordillera, Guairá, Paraguarí, San Pedro.

Voucher: F. Mereles 3983 (FCQ).

Sida rhombifolia L., Sp. Pl. 2: 684. 1753.

Syn.: Sida rhombifolia L. var. canariensis (Willd.) Griseb., S. rhombifolia var. rhomboidea (Roxb.) Mast., S. rhomboidea Roxb., S. subrhombiformis Larrańaga

Herb or subshrub.

Departmental distribution: Alto Paraguay, Amambay, Boquerón, Caaguazú, Central, Cordillera, Guairá, Itapúa, Neembucú, Paraguarí, Presidente Hayes, San Pedro. Voucher: J. De Egea et al. 1231(FCQ).

Sida santaremensis Monteiro, Monogr. Malv. Brasil. Fasc. I. 44. 1936.

Syn.: Sida glaziovii auct. non K.Schum., S. rhombifolia L. var. subtomentosa K.Schum., S. santaremensis Monteiro var. krapovickasiana Monteiro

Subshrub.

Departmental distribution: Alto Paraguay, Amambay, Boquerón, Caaguazú, Central, Concepción, Cordillera, Guairá, Paraguarí, Presidente Hayes, San Pedro.

Voucher: N. Soria 1234 (FCQ).

Sida spinosa L., Sp. Pl. 2: 683. 1753.

Syn.: Sida angustifolia Lam., S. escobilla Larrańaga, S. spinosa L. var. angustifolia (Lam.) Griseb.

Subshrub.

Departmental distribution: Alto Paraguay, Boquerón, Central, Cordillera, Guairá, Paraguarí, Presidente Hayes.

Voucher: E. Bordas 4198 (FCQ). 
Sidastrum paniculatum (L.) Fryxell, Brittonia 30(4): 453. 1978.

Syn.: Sida paniculata L.

Subshrub.

Departmental distribution: Alto Paraguay, Caaguazú, Canindeyú, Central, Concepción, Cordillera, Guairá, Misiones, Neembucú, Paraguarí, Presidente Hayes, San Pedro.

Voucher: G. Schmeda 314 (FCQ).

Triumfetta semitriloba Jacq., Enum. Syst. Pl. 22. 1760.

Syn.: Triumfetta abutiloides A.St.-Hil., T. tricuspis A.St.-Hil.

Shrub.

Departmental distribution: Alto Paraná, Amambay, Caaguazú, Caazapá, Canindeyú, Central, Concepción, Cordillera, Guairá, Itapúa, Misiones, Neembucú, Paraguarí, San Pedro.

Voucher: I. Basualdo 818 (FCQ).

Waltheria indica L., Sp. Pl. 2: 673. 1753.

Syn.: Waltheria americana L., W. americana var. indica (L.) K.Schum.

Subshrub.

Departmental distribution: Alto Paraguay, Alto Paraná, Amambay, Boquerón, Caaguazú, Canindeyú, Central, Concepción, Cordillera, Guairá, Itapúa, Misiones, Paraguarí, Presidente Hayes, San Pedro.

Voucher: I. Basualdo 179 (FCQ).

Wissadula densiflora R.E.Fr. var. densiflora, Kungl. Svenska Vetenskapsakad. Handl. 43(4): 64, pl. 4, 6. 1908.

Subshrub.

Departmental distribution: Alto Paraguay, Boquerón, Presidente Hayes.

Voucher: F. Mereles \& S. Soria 9948 (BM, FCQ, G). 
Wissadula subpeltata (Kuntze) R.E.Fr., Kungl. Svenska Vetenskapsakad. Handl. 43(4): 56, pl. 5, 6, 7. 1908.

Syn.: Abutilon amplissimum (L.) Kuntze var. subpeltatum Kuntze, Wissadula amplissima auct. non (L.) R.E.Fr.

Shrub.

Departmental distribution: Alto Paraguay, Alto Paraná, Amambay, Caaguazú, Concepción, Cordillera, Guairá, Paraguarí, Presidente Hayes.

Voucher: M. Dematteis et al. 2788 (FCQ).

\section{MOLLUGINACEAE}

Mollugo verticillata L., Sp. Pl. 1: 89. 1753.

Syn.: Mollugo arenaria Kunth

Herb.

Departmental distribution: Alto Paraguay, Amambay, Boquerón, Caazapá, Central, Concepción, Cordillera, Paraguarí, Presidente Hayes.

Voucher: G. Céspedes 424 (FCQ).

\section{MORACEAE}

Dorstenia brasiliensis Lam., Encycl. 2(1): 317. 1786.

Syn.: D. brasiliensis Lam. f. balansae Chodat, D. brasiliensis var. guaranitica Chodat, hom. illeg., D. brasiliensis var. major Chodat \& Hassl., D. brasiliensis var. palustris Hassl., D. brasiliensis var. tomentosa (Fisch. \& C.A.Mey.) Hassl., D. brasiliensis var. tubicina (Ruiz \& Pav.) Chodat \& Vischer D. brasiliensis var. typica Hassl., nom. inval., D. montevidensis Field \& Gardner, Dorstenia schulzii Carauta, C.Valente \& Dunn de Araujo, D. tomentosa Fisch. \& C.A.Mey., D. tubicina Ruiz \& Pav., D. tubicina var. genuina Hassl., nom. inval., D. tubicina f. major Hassl., D. tubicina var. opifera (Mart.) Hassl. D. tubicina f. subexcentrica Hassl., D. tubicina f. typica Hassl., nom. inval.

Herb.

Departmental distribution: Alto Paraguay, Amambay, Caaguazú, Central, Cordillera, Guairá, Neembucú, Presidente Hayes, San Pedro.

Voucher: J. De Egea et al. 1255 (FCQ). 


\section{NYCTAGINACEAE}

Boerhavia diffusa L. var. diffusa, Sp. Pl. 1: 3. 1753.

Syn.: Boerhavia coccinea Mill. var. paniculata (Rich.) Moscoso, B. paniculata Rich.

Herb. Introduced.

Departmental distribution: Alto Paraguay, Boquerón, Caazapá, Central, Guairá, Presidente Hayes.

Voucher: F. Mereles \& S. Soria 9985 (BM, FCQ, G).

Boerhavia diffusa L. var. leiocarpha (Heimer) C.D.Adams, Mitt. Bot. Staatssamml. München 8: 115. 1970.

Syn.: Boerhavia ciliato-bracteata Heimerl, B. coccinea Mill. var. leiocarpa (Heimerl) Standl., B. diffusa L. var. paniculata D.Parodi, nom. nud., B. friesii Heimerl, $B$. paniculata Rich. var. guaranitica Heimerl, B. paniculata var. leiocarpa Heimerl, $B$. paniculata f. leiocarpa Heimerl

Herb. Introduced.

Departmental distribution: Alto Paraguay, Cordillera, Presidente Hayes. Voucher: F. Mereles \& S. Soria 10184 (FCQ).

\section{ONAGRACEAE}

Ludwigia lagunae (Morong) H.Hara, J. Jap. Bot. 28: 292. 1953.

Syn.: Jussiaea brachycarpa Lam. ssp. epilobioides (Chodat \& Hassl.) Hassl., J. brachycarpa var. genuina Hassl., nom. inval., J. brachycarpa var. grandiflora Hassl., J. brachycarpa var. paraguayensis (Chodat) Hassl., J. brachycarpa var. parviflora (Chodat \& Hassl.) Hassl., J. brachycarpa var. puberula Hassl., J. brachycarpa Micheli, hom. illeg., J. epilobioides Chodat \& Hassl., J. epilobioides var. parviflora Chodat \& Hassl., J. lagunae Morong, J. lagunae var. paraguayensis (Chodat) Munz, J. lagunae var. typica Munz, nom. inval., J. leveilleana Bertoni, J. paraguayensis Chodat, J. suffruticosa L. var. epilobioides (Chodat \& Hassl.) Bertoni, J. suffruticosa var. paraguayensis (Chodat) Bertoni, J. suffruticosa var. parviflora (Chodat \& Hassl.) Bertoni

Herb or subshrub.

Departmental distribution: Alto Paraguay, Amambay, Caaguazú, Canindeyú, Central, Concepción, Cordillera, Guairá, Misiones, Neembucú, Paraguarí, Presidente Hayes, San Pedro.

Voucher: F. Mereles \& J. De Egea 10151 (FCQ, G). 


\section{OXALIDACEAE}

Oxalis conorrhiza Jacq., Monographia, Iconibus Illustrata 26. 1794.

Syn.: Acetosella caespitosa (A.St.-Hil.) Kuntze, A. chrysantha (Progel) Kuntze, A. cineracea (A.St.-Hil.) Kuntze, $A$. commersonii (Pers.) Kuntze, $A$. conorrhiza (Jacq.) Kuntze, A. megapotamica (Spreng.) Kuntze, Oxalis andicola Gillies ex Hook. \& Arn., O. andicola var. wallichiana Gillies ex Hook. \& Arn., O. brevipes Fredr., O. caespitosa A.St.-Hil., O. chrysantha Progel, O. chrysantha var. pusilla Progel, O. cineracea A.St.-Hil., O. commersonii Pers., O. conorhixa Larrañaga, O. cordobensis R.Knuth, O. cordobensis var. humilior R.Knuth, O. corniculata L. var. serpens R.Knuth, O. hassleriana Chodat, O. linneaformis R. Knuth, O. megapotamica Spreng., O. repens Thunb. f. uniflora Hieron. \& Lorentz ex R.Knuth, nom. nud., O. sexenata Savigny, O. sternbergii auct. non Zucc., Xanthoxalis chrysantha (Progel) Holub, X. commersonii (Pers.) Holub, X. cordobensis (R.Knuth) Holub

Herb.

Departmental distribution: Alto Paraná, Alto Paraguay, Amambay, Caaguazú, Central, Concepción, Cordillera, Misiones, Presidente Hayes.

Voucher: F. Mereles et al. 8100 (FCQ).

Oxalis corniculata L. var. corniculata., Sp. Pl. 1: 435. 1753.

Syn.: Acetosella corniculata (L.) Kuntze, A. corniculata var. repens (Thunb.) Kuntze, Oxalis steudeliana R.Knuth, Xanthoxalis corniculata (L.) Small

Herb.

Departmental distribution: Alto Paraná, Central, Cordillera, Guairá. Voucher: E. Zardini 23445 (AS, MO).

Oxalis paludosa A.St.-Hil., Fl. Bras. Merid. 1: 121. 1825.

Syn.: Acetosella montevidensis (Progel) Kuntze, A. paludosa (A.St.-Hil.) Kuntze, Oxalis corrientesensis R.Knuth, O. duricaulis R.Knuth, O. montevidensis Progel

Herb.

Departmental distribution: Central, Cordillera, Neembucú, Paraguarí, Presidente Hayes.

Voucher: G. Céspedes 438 (FCQ). 


\section{PASSIFLORACEAE}

Turnera grandidentata (Urb.) Arbo, Candollea 40(1): 176. 1985.

Syn.: Turnera ulmifolia L. var. elegans auct. non (Otto) Urb., T. ulmifolia L. var. grandidentata Urb.

Herb. Endemic.

Departmental distribution: Caaguazú, Central, Concepción, Cordillera, Misiones, Paraguarí, San Pedro.

Voucher: F. González \& M. J. López 554 (FCQ).

\section{PHYLLANTHACEAE}

Phyllanthus niruri L., Sp. Pl. 2: 981. 1753.

Syn.: Phyllanthus lathyroides Kunth, P. lathyroides f. rosellus Müll.Arg., P. niruri L. ssp. lathyroides (Kunth) G.L.Webster, P. microphyllus Mart., P. niruri f. microphyllus (Müll.Arg.) G.L.Webster, P. rosellus (Müll.Arg.) Müll.Arg.

Herb.

Departmental distribution: Caazapá, Canindeyú, Central, Concepción, Cordillera, Guairá, Itapúa, Neembucú, Paraguarí.

Voucher: E. Zardini et al. 2421 (FCQ, MO).

Phyllanthus orbiculatus Rich., Actes Soc. Hist. Nat. Paris 1:113. 1792.

Syn.: Orbicularia orbiculata (Rich.) Moldenke

Herb.

Departmental distribution: Alto Paraná, Amambay, Canindeyú, Central, Concepción, Cordillera, Guairá, Paraguarí.

Voucher: E. Zardini \& R. Degen 3767 (FCQ, MO).

\section{PLANTAGINACEAE}

Plantago tomentosa Lam. ssp. tomentosa, Tabl. Encycl. 1(2): 340. 1792.

Syn.: Plantago achalensis Pilg., P. achalensis var. hirtula Pilg., P. achalensis f. minor Pilg., $P$. affinis Decne., $P$. arechavaletai Pilg., $P$. bicallosa Decne. var. hirsutior Pilg., $P$. 
grisebachii Hieron., P. hypolasia Pilg., P. hypoleuca Pilg., P. oreades Decne. var. lanuginosa Griseb, P. paralias Decne., P. paralias var. achalensis (Pilg.) Pilg., P. paralias ssp. affinis (Decne.) Pilg., P. paralias var. brevifolia Pilg., P. paralias var. cordobensis (Pilg.) Pilg., P. paralias ssp. dasystachys (Pilg.) Pilg., P. paralias ssp. euparalias Pilg., nom. inval., P. paralias var. glabrescens (Pilg.) Pilg, P. paralias ssp. grisebachii (Hieron.) Pilg., P. paralias ssp. hypolasia (Pilg.) Pilg., P. paralias var. lasiophylla (Pilg.) Pilg, P. paralias ssp. leiocalyx (Pilg.) Pilg., P. paralias var. mollior (Pilg.) Pilg, P. paralias ssp. petiolata (Pilg.) Pilg., P. paralias var. saxicola (Pilg.) Pilg, P. paralias ssp. schlechtendaliana (Pilg.) Pilg., P. paralias ssp. selloana (Pilg.) Pilg., P. tomentosa var. achalensis Pilg., P. tomentosa ssp. balansai Pilg., P. tomentosa var. brevifolia Pilg., P. tomentosa var. cordobensis Pilg., P. tomentosa ssp. dasystachys Pilg., P. tomentosa var. glabrescens Schmidt, P. tomentosa Lam. ssp. grisebachii (Hieron.) Pilg., P. tomentosa ssp. hypolasia (Pilg.) Pilg., P. tomentosa var. lasiophylla Pilg., P. tomentosa ssp. leiocalyx Pilg., P. tomentosa var. mollior Pilg., P. tomentosa ssp. paralias (Decne.) Pilg., P. tomentosa ssp. petiolata Pilg., P. tomentosa var. saxicola Pilg, P. tomentosa ssp. schlechtendaliana Pilg., P. tomentosa ssp. selloana Pilg.

Herb.

Departmental distribution: Alto Paraguay, Caaguazú, Caazapá, Canindeyú, Central, Cordillera, Guairá, Neembucú, Paraguarí, Presidente Hayes, SanPedro

Voucher: R. Degen et al. 4348 (FCQ).

Scoparia dulcis L., Sp. Pl. 1: 116. 1753.

Syn.: Capraria dulcis (L.) Kuntze, Scoparia dulcis L. var. tenuifolia Griseb., S. nudicaulis Chodat \& Hassl., S. procumbens Jacq., S. purpurea Ridl.

Herb or subshrub.

Departmental distribution: Alto Paraguay, Alto Paraná, Amambay, Caaguazú, Caazapá, Canindeyú, Central, Concepción, Cordillera, Guairá, Misiones, Neembucú, Paraguarí, Presidente Hayes, San Pedro.

Voucher: F. Mereles et al. 10072 (FCQ).

Scoparia ericacea Cham. \& Schltdl., Linnaea 2: 604. 1827.

Syn.: Capraria ericacea (Cham. \& Schltdl.) Kuntze, Scoparia divaricata R.E.Fr., S. plebeja Cham. \& Schltdl.

Herb or subshrub.

Departmental distribution: Alto Paraná, Cordillera, Presidente Hayes, San Pedro.

Voucher: F. Mereles et al. 10071 (FCQ). 
Stemodia verticillata (Mill.) Hassl., Trab. Mus. Farmacol. 21: 110. 1909.

Syn.: Capraria humilis Sol., Erinus verticillatus Mill., Lendneria humilis (Sol.) Minod, L. verticillata (Mill.) Britton, Stemodia arenaria Kunth, Stemodia humilis (Sol.) G. Dawson, Stemodia macrotricha Colla, Stemodia parviflora W.T.Aiton, Stemodiacra verticillata (Mill.) Kuntze

Herb.

Departmental distribution: Caazapá, Canindeyú, Cordillera, Guairá, Ñeembucú, San Pedro.

Voucher: J. De Egea et al. 1220 (BM, FCQ).

\section{POACEAE}

Andropogon bicornis L., Sp. Pl. 2: 1046. 1753.

Syn.: Andropogon bicornis L. var. absconditus Hack., A. bicornis var. burchellii Hack., A. bicornis var. gracillimus Hack., A. bicornis var. virginicoides Hack.

Herb.

Departmental distribution: Alto Paraná, Amambay, Canindeyú, Central, Guairá, Itapúa, Misiones, Neembucú, Paraguarí, Presidente Hayes, San Pedro.

Voucher: F. Mereles et al. 10179 (FCQ, G).

Avena sativa L. subsp. sativa, Sp.Pl. 1: 79. 1753.

Syn.: Avena fatua L. f. glaberrima Thell., A. fatua ssp. sativa (L.) Thell., A. sativa L. var. glaberrima (Thell.) Parodi

Herb. Introduced.

Departmental distribution: Alto Paraná.

Voucher: F. Mereles et al. 10074 (FCQ).

Cenchrus ciliaris L., Mant. Pl. 2: 302. 1771.

Syn.: Pennisetum cenchroides Rich. ex Pers., nom. illeg., P. ciliare (L.) Link

Herb. Introduced.

Departmental distribution: Alto Paraguay, Boquerón, Guairá, Presidente Hayes.

Voucher: F. Mereles \& S. Soria 9969 (FCQ, G). 
Cenchrus echinatus L., Sp. Pl. 2: 1050. 1753.

Syn.: Cenchrus crinitus Mez, C. echinatus Cav., nom. inval.

Herb.

Departmental distribution: Alto Paraguay, Alto Paraná, Central, Concepción, Cordillera, Guairá, Itapúa, Paraguarí, Presidente Hayes, San Pedro.

Voucher: J. De Egea et al. 1293 (FCQ).

Cenchrus purpureus (Schumach.) Morrone, Ann. Bot. (Oxford) 106(1): 129. 2010.

Syn.: Pennisetum purpureum Schumach.

Herb. Intruduced.

Departmental distribution: Alto Paraná, Canindeyú, Central, Paraguarí.

Voucher: F. Mereles et al. 10083 (BM, FCQ, G).

Chloris virgata Sw., Fl. Ind. Occid. 1: 203. 1797.

Syn.: Chloris compressa DC., C. elegans Kunth

Herb.

Departmental distribution: Alto Paraguay, Boquerón.

Voucher: F. Mereles \& S. Soria 10195 (FCQ).

Cynodon dactylon (L.) Pers. var. dactylon, Syn. Pl. 1: 85. 1805.

Syn.: Capriola dactylon (L.) Kuntze, Cynodon aristiglumis Caro \& E.A.Sánchez, Cynodon aristulatus Caro \& E.A.Sánchez, Cynodon erectus J.Presl., Cynodon pascurus Nees, Panicum dactyon $\mathrm{L}$.

Herb. Introduced.

Departmental distribution: Alto Paraná, Cordillera, Guairá, Itapúa, San Pedro.

Voucher: J. De Egea et al. 1304 (FCQ).

Cynodon dactylon (L.) Pers. var. longiglumis Caro \& E.A.Sánchez, Kurtziana 5: 210, f. 2G. 1969.

Herb. 
Departmental distribution: Itapúa, San Pedro.

Voucher: J. De Egea et al. 1282 (FCQ).

Digitaria aequiglumis (Hack. \& Arechav.) Parodi var. aequiglumis, Revista Fac. Agron. Veterin. 4: 47. 1922.

Syn.: Digitaria campestris Henrard, D. chillanensis Phil. ex Henrard, nom. nud., Panicum aequiglume Hack. \& Arechav., P. debile Phil., nom. illeg., P. debile Desf. var. aequiglume (Hack. \& Arechav.) Hack., P. ramosum Arechav., P. tridactylum Phil., hom. illeg., Syntherisma aequiglumis (Hack. \& Arechav.) Hitchc.

Herb.

Departmental distribution: Alto Paraná, Central, Itapúa, Presidente Hayes.

Voucher: C.V.M. Pavetti 10974 (Zuloaga et al. 1994).

Digitaria balansae Henrard, Meded. Rijks-Herb. 61: 2. 1930.

Herb.

Departmental distribution: Alto Paraná, Caaguazú, Caazapá, Cordillera, Guairá, Itapúa, Misiones, Paraguarí, Presidente Hayes.

Voucher: T. Rojas 11188 (AS, MO).

Digitaria bicornis (Lam.) Roem. \& Schult., Syst. Veg. (ed. 15 bis) 2: 470. 1817.

Syn.: Paspalum bicorne Lam., Panicum bicorne (Lam.) Kunth, Panicum glaucescens Nees, hom. illeg.

Herb. Introduced.

Departmental distribution: Alto Paraguay, Alto Paraná, Amambay, Boquerón, Central, Concepción, Cordillera, Itapúa, Misiones, Presidente Hayes, San Pedro. Voucher: F. Mereles et al. 9918 (FCQ).

Digitaria ciliaris (Retz.) Koeler, Descr. Gram. 27. 1802.

Syn.: Digitaria abortiva Reeder, D. adscendens (Kunth) Henrard, D. marginata Link, D. tarapacana Phil., Panicum adscendes Kunth, P. ciliare Retz., P. sanguinale L. var. ciliare (Retz.) Vasey

Herb. 
Departmental distribution: Alto Paraguay, Alto Paraná, Amambay, Caaguazú, Canindeyú, Central, Concepción, Cordillera, Guairá, Itapúa, Misiones, Paraguarí, Presidente Hayes, San Pedro.

Voucher: F. Mereles et al. 10069 (FCQ).

Digitaria horizontalis Willd., Enum. Pl. 92. 1809.

Syn.: Panicum horizontale (Willd.) G.Mey., P. oxyanthum Steud., P. sanguinale L. var. horizontale (Willd.) Schweinf.

Herb.

Departmental distribution: Alto Paraguay, Amambay, Boquerón, Canindeyú, Central, Concepción, Cordillera, Guairá, Itapúa, Misiones, Presidente Hayes, San Pedro. Voucher: W.J. Hahn 2076 (MO, PY).

Digitaria insularis (L.) Fedde, Bot. Jahresber. 31(1): 778. 1904.

Syn.: Acicarpa sacchariflora Raddi, nom. illeg., Andropogon insulare L., Digitaria insularis (L.) Mez ex Ekman, comb. superfl., Panicum insulare (L.) G.Mey., P. insulare var. typicum Hack., nom. inval., P. lanatum Rottb., nom. superfl., P. leucophaeum Kunth, Milium villosum Sw., nom. superfl., Trichachne insularis (L.) Nees, Tricholaena insularis (L.) Griseb., Syntherisma insularis (L.) Millsp., Valota insularis (L.) Chase.

Herb.

Departmental distribution: Alto Paraguay, Alto Paraná, Amambay, Boquerón, Caaguazú, Central, Cordillera, Guairá, Misiones, Paraguarí, Presidente Hayes, San Pedro.

Voucher: F. Mereles \& S. Soria 9989 (BM, FCQ, G).

Echinochloa colona (L.) Link., Hort. Berol. 2: 209. 1833.

Syn.: Echinochloa colona (L.) Link f. zonalis (Guss.) Wiegand, Panicum colonum L., P. colonum f. maculatum Arechav., Oplismenus colonus (L.) Kunth, O. muticus Phil.

Herb.

Departmental distribution: Alto Paraguay, Amambay, Central, Concepción, Cordillera, Guairá, Itapúa, Misiones, Presidente Hayes.

Voucher: F. Mereles et al. 10165 (BM, FCQ, G). 
Echinochloa crus-galli (L.) P.Beauv. var. crus-galli, Ess. Agrostogr. 161 (53, 169). 1812.

Syn.: Panicum crus-galli L.

Herb. Introduced.

Departmental distribution: Alto Paraguay, Central, Cordillera, Presidente Hayes.

Voucher: F. Mereles \& L. Ramella 2925 (FCQ).

Echinochloa crus-pavonis (Kunth) Schult., Mant. 2: 269. 1824.

Syn.: Echinochloa crus-galli (L.) P.Beauv. var. crus-pavonis (Kunth) Hitchc., Oplismenus crus-pavonis Kunth, Panicum crus-galli L. var. sabulicola (Nees) Trin.

Herb. Introduced.

Departmental distribution: Alto Paraguay, Alto Paraná, Boquerón, Central, Concepción, Cordillera, Guairá, Itapúa, Misiones, Presidente Hayes.

Voucher: F. Mereles \& J. De Egea 10135 (BM, FCQ, G).

Echinochloa oryzoides (Ard.) Fritsch, Verh. K.K. Zool.-Bot. Ges. Wien 41: 742. 1891.

Syn.: Panicum crus-galli L. var. grandiflorum Döll, P. oryzoides Ard.

Herb. Introduced.

Departmental distribution: Cordillera.

Voucher: E. Lurvey 60 (Zuloaga et al. 1994).

Echinochloa polystachya (Kunth) Hitchc. var. spectabilis (Nees) Mart.Crov., Revista Argent. Agron. 9: 318. 1942.

Syn.: Echinochloa spectabilis (Nees ex Trin.) Link, Panicum spectabile Nees ex Trin., Pseudoechinolaena spectabilis (Nees ex Trin.) Herter

Herb.

Departmental distribution: Alto Paraguay, Central, Cordillera, Guairá, Neembucú, Presidente Hayes.

Voucher: J. De Egea et al. 1311 (FCQ). 
Eleusine indica (L.) Gaertn., Fruct. Sem. Pl. 1: 8. 1788.

Syn.: Cynosurus indicus L.

Herb. Introduced.

Departmental distribution: Amambay, Caazapá, Central, Guairá, Itapúa, Misiones. Voucher: F. Mereles et al. 10162 (FCQ, G).

Eleusine tristachya (Lam.) Lam., Tabl. Encycl. 1(2): 203. 1792.

Syn.: Cynosurus tristachyos Lam., Eleusine indica (L.) Gaertn. var. condensata Döll, Eleusine oligostachya Link, E. tristachya (Lam.) Kunth, comb. superfl.

Herb.

Departmental distribution: Alto Paraná, Central, Guairá, Itapúa, Misiones, Neembucú, Misiones, Paraguarí, Presidente Hayes, San Pedro.

Voucher: J. De Egea et al. 1292 (FCQ).

Eragrostis airoides Nees, Fl. Bras. Enum. Pl. 2(1): 509. 1829.

Syn.: Agrosticula brasiliensis (Raddi) Herter, Aira brasiliensis Raddi, Airopsis millegrana Griseb., Eragrostis triflora Ekman, Poa airoides (Nees) Kunth, hom. illeg., Sporobolus brasiliensis (Raddi) Hack.

Herb.

Departmental distribution: Alto Paraná, Amambay, Central, Guairá, Itapúa, Misiones, Paraguarí, Presidente Hayes, San Pedro.

Voucher: G. Céspedes et al. 440 (FCQ).

Eragrostis bahiensis Schrad., Mant. 2: 318. 1824.

Syn.: Eragrostis atrovirens auct. non (Desf.) Trin., E. bahiensis Schrad. ex Schult. var. contracta Döll, E. bahiensis var. laxiuscula Döll, E. bahiensis f. riparia Burkart, E. blepharophylla Jedwabn., E. expansa Link, E. firma Trin., E. macra Jedwabn., E. microstachya (Link) Link, E. pilosa (L.) P.Beauv. var. bahiensis (Schrad. ex Schult.) Kuntze, E. psammodes Trin., E. psammodes var. microstachya (Link) Döll, E. squamata auct. non (Lam.) Steud., Poa expansa (Link) Kunth, P. microstachya Link, P. psammodes (Trin.) Kunth. 
Herb.

Departmental distribution: Alto Paraná, Amambay, Caaguazú, Central, Concepción, Cordillera, Guairá, Misiones, Paraguarí, Presidente Hayes, San Pedro.

Voucher: G. Céspedes et al. 448 (FCQ).

Eragrostis lugens Nees, Fl. Bras. Enum. Pl. 2(1): 507. 1829.

Syn.: Eragrostis flaccida Lindm., E. lugens Nees ssp. flaccida (Lindm.) Hack., E. lugens var. glabrata Döll, E. lugens f. pallida Hack., E. pilosa (L.) P.Beauv. var. lugens (Nees) Griseb., Poa lugens (Nees) Kunth

Herb.

Departmental distribution: Alto Paraguay, Boquerón, Central, Concepción, Cordillera, Guairá, Neembucú, Paraguarí, Presidente Hayes.

Voucher: G. Céspedes 437 (FCQ).

Eragrostis pilosa (L.) P.Beauv., Ess. Agrostogr. 71 (162, 175). 1812.

Syn.: Eragrostis damiensiana (Bonnet) Thell., E. damiensiana var. condensata (Hack.) Thell., E. gracilis Schrad., E. multicaulis Steud., E. peregrina Wiegand, E. pilosa (L.) P.Beauv. var. condensata Hack., E. pilosa ssp. damiensiana (Bonnet) Thell., E. pilosa var. damiensiana Bonnet, E. verticillata (Cav.) P.Beauv., Poa pilosa L.

Herb. Introduced.

Departmental distribution: Alto Paraná, Central, Cordillera.

Voucher: M. Ortiz 2 (FCQ).

Hymenachne amplexicaulis (Rudge) Nees, Fl. Bras. Enum. Pl. 2(1): 276. 1829.

Syn.: Agrostis monostachya Poir., Panicum amplexicaule Rudge

Herb.

Departmental distribution: Alto Paraguay, Alto Paraná, Amambay, Boquerón, Caaguazú, Caazapá, Central, Concepción, Cordillera, Guairá, Itapúa, Misiones, Neembucú, Paraguarí, Presidente Hayes, San Pedro.

Voucher: F. Mereles et al. 10180 (FCQ). 
Leptochloa virgata (L.) P.Beauv., Ess. Agrostogr. 71 (161, 166). 1812.

Syn.: Cynodon domingense (Jacq.) Raspail, Cynodon virgatus (L.) Raspail, Cynosurus dominguensis Jacq., Cynosurus virgatus L., Eleusine barbata Desv., E. domingensis (Jacq.) Pers., E. unioloides Willd. ex Steud., nom. inval., E. virgata (L.) Pers., Diplachne domingensis (Jacq.) Chapm., Festuca domingensis (Jacq.) Lam., F. virgata (L.) Lam., Leptochloa barbata (Desv.) Parodi ex Nicora, Leptochloa domingensis (Jacq.) Trin., Leptochloa mutica Steud., Leptochloa procera Nees, Leptochloa virgata (Jacq.) Chapm. var. domingensis (Jacq.) Link ex Griseb., Leptochloa virgata (L.) P.Beauv. var. mutica (Steud.) Döll, Leptochloa virgata (L.) P.Beauv. var. puberula Hack, Leptostachys domingensis (Jacq.) G.Mey.Rabdochloa domingensis (Jacq.) P.Beauv.

Herb.

Departmental distribution: Alto Paraguay, Alto Paraná, Amambay, Caaguazú, Caazapá, Central, Concepción, Cordillera, Guairá, Itapúa, Misiones, Paraguarí, Presidente Hayes, San Pedro.

Voucher: D.R. Brunner 1219 (MO, PY).

Megathyrsus maximus (Jacq.) B.K.Simon \& S.W.L.Jacob var. maximus, Austrobaileya 6(3): 572. 2003.

Syn.: Panicum maximum Jacq., Urochloa maxima (Jacq.) R.D.Webster

Herb. Introduced.

Departmental distribution: Alto Paraguay, Alto Paraná, Amambay, Boquerón, Caazapá, Central, Concepción, Cordillera, Guairá, Itapúa, Paraguarí, Presidente Hayes, San Pedro.

Voucher: R. Degen 1518 (FCQ).

Melinis repens (Willd.) Zizka, Biblioth. Bot. 138: 55. 1988.

Syn.: Rhynchelytrum repens (Willd.) C.E.Hubb., R. roseum (Nees) Stapf \& C.E.Hubb., Saccharum repens Willd., Tricholaena repens (Willd.) Hitchc.

Herb. Introduced.

Departmental distribution: Alto Paraguay, Alto Paraná, Amambay, Canindeyú, Central, Concepción, Cordillera, Guairá, Itapúa, Misiones, Neembucú, Paraguarí, Presidente Hayes, San Pedro.

Voucher: J. De Egea et al. 1271 (FCQ). 
Panicum bergii Arechav. var. bergii, Anales Mus. Nac. Montevideo 1: 147. 1894.

Syn.: Panicum bergii Arechav. f. convoluta R.A.Palacios, P. burkartii Zuloaga, P. pilcomayense Hack.

Herb.

Departmental distribution: Alto Paraguay, Alto Paraná, Boquerón, Central, Concepción, Misiones, Neembucú, Paraguarí, Presidente Hayes.

Voucher: F. Mereles et al. 10085 (FCQ, G).

Paspalum acuminatum Raddi, Agrostogr. Bras. 25. 1823.

Syn.: Paspalum serratum Hitchc. \& Chase

Herb.

Departmental distribution: Amambay, Caazapá, Central, Concepción, Cordillera, Itapúa. Voucher: A.Schinini 6282 (CTES,G).

Paspalum almum Chase, J. Wash. Acad. Sci. 23(3): 137, f. 1. 1933.

Syn.: Paspalum hexastachyum Parodi, P. ovale Nees ex Steud. var. apiculatum Hack.

Herb.

Departmental distribution: Alto Paraguay, Alto Paraná, Central, Cordillera, Misiones, Neembucú, Paraguarí, Presidente Hayes.

Voucher: M. Peña-Chocarro et al. 1669 (FCQ).

Paspalum chaseanum Parodi, J. Wash. Acad. Sci. 23: 137. 1933.

Herb.

Departmental distribution: Alto Paraguay, Boquerón.

Voucher: F. Mereles \& R. Degen 5885 (FCQ).

Paspalum conjugatum P.J.Bergius, Acta Helv. Phys.-Math. 7: 129 (130; t. 8). 1772.

Syn.: Paspalum renggeri Steud.

Herb. 
Departmental distribution: Alto Paraná, Amambay, Caaguazú, Caazapá, Canindeyú, Central, Concepción, Cordillera, Guairá, Misiones, Paraguarí, San Pedro. Voucher: O. Morrone \& J. Pensiero 133 (FCQ).

Paspalum plicatulum Michx., Fl. Bor.-Amer. 1: 45. 1803.

Syn.: Panicum plicatulum (Michx.) Kuntze, Paspalum lenticulare Kunth f. intumescens (Döll) Killeen, Paspalum montevidense Spreng., Paspalum plicatulum Michx. var. genuinum Parodi, nom. inval., Paspalum plicatulum var. glabrum Arechav., Paspalum plicatulum var. intumescens Döll, Paspalum plicatulum var. longipilum Hack., P. plicatulum ssp. montevidense (Spreng.) Roseng., B.R.Arrill. \& Izag., Paspalum plicatulum var. villosissimum Pilg., P. ramosum Swallen

Herb.

Departmental distribution: Alto Paraguay, Alto Paraná, Amambay, Caaguazú, Central, Concepción, Cordillera, Guairá, Itapúa, Misiones, Neembucú, Paraguarí, Presidente Hayes, San Pedro.

Voucher: J. De Egea et al. 1308 (FCQ).

Paspalum umbrosum Trin., Mém. Acad. Imp. Sci. St.-Pétersbourg, Sér. 6, Sci. Math. 1: 153.1834.

Syn.: Paspalum densiflorum Döll, P. maritimum auct. non Trin., P. paniculatum L. ssp. umbrosum (Trin.) Roseng., B.R.Arrill. \& Izag.

Herb.

Departmental distribution: Alto Paraná, Caaguazú, Caazapá, Central, Cordillera, Guairá, Itapúa, Misiones, Paraguarí, San Pedro.

Voucher: F. Mereles et al. 10093 (FCQ).

Paspalum urvillei Steud., Syn. Pl. Glumac. 1(1): 24. 1853.

Syn.: Paspalum larrañagai Arechav.

Herb.

Departmental distribution: Alto Paraná, Amambay, Caaguazú, Caazapá, Canindeyú, Central, Concepción, Cordillera, Guairá, Itapúa, Misiones, Neembucú, Paraguarí, San Pedro.

Voucher: E. Lurvey 72 ( Zuloaga et al. 2014). 
Paspalum wrightii Hitchc. \& Chase, Contr. U.S. Natl. Herb. 18: 310. 1917.

Syn.: Paspalum hydrophilum Henrard, P. plicatulum Michx. var. multinode Hack, $P$. virgatum L. var. subplicatum Hack

Herb.

Departmental distribution: Alto Paraguay, Central, Neembucú, Presidente Hayes.

Voucher: J.R. Ramirez 132 (CTES).

Setaria parviflora (Poir.) Kerguélen var. parviflora, Lejeunia 120: 161. 1987.

Syn.: For full list of synonyms see Pensiero (2003).

Herb.

Departmental distribution: Alto Paraguay, Alto Paraná, Amambay, Caaguazú, Canindeyú, Central, Concepción, Cordillera, Guairá, Itapúa, Paraguarí, Presidente Hayes, San Pedro.

Voucher: F. Mereles et al. 10100 (FCQ).

Setaria scandens Schrad., Mant. 2: 279. 1824.

Syn.: Chaetochloa scandens (Schrad.) Scribn., Panicum scandens (Schrad.) Trin., P. scandens var. grandiflorum Döll, Panicum scandens var. longiseta Döll, P. scandens var. vulgare Döll, Setaria scandens Schrad. ex Schult. var. sphacelata Hack., S. trinii Kunth.

Herb.

Departmental distribution: Amambay, Guairá.

Voucher: E. Hassler \& T. Rojas 10141 (Zuloaga et al. 2014).

Setaria tenax (Rich.) Desv., Opusc. Sci. Phys. Nat. 78. 1831.

Syn.: Panicum tenax A.Rich., Setaria sphaerocarpa (Salzm.) C.E.Hubb.

Herb.

Departmental distribution: Central, Concepción, Cordillera, Paraguarí.

Voucher: E.M. Zardini \& C. Velázquez 26273 (FCQ, MO). 
Sorghum halepense (L.) Pers. var. halepense, Syn. Pl. 1: 101. 1805.

Syn.: Andropogon halepensis (L.) Brot., A. sorghum (L.) Brot. var. halepensis (L.) Hack., Holcus halepensis L., Sorghum halepense (L.) Pers. var. genuinum Hack., nom. inval.

Herb. Introduced.

Departmental distribution: Alto Paraná, Boquerón, Central, Itapúa.

Voucher: F. Mereles et al. 10084 (FCQ).

Sporobolus pyramidatus (Lam.) Hitchc., Man. Grasses W. Ind. 84. 1936.

Syn.: Agrostis pyramidata Lam., Sporobolus argutus (Nees) Kunth, S. argutus f. purpurascens Hack., S. argutus var. tuberculatus (Hack.) Hack., S. tuberculatus Hack., Vilfa arguta Nees

Herb.

Departmental distribution: Alto Paraguay, Boquerón, Paraguarí, Presidente Hayes.

Voucher: F. Mereles \& S. Soria 9970 (BM, CTES, FCQ, G).

Stapfochloa elata (Desv.) P.M.Peterson, Taxon 64: 460. 2015.

Syn.: Andropogon barbatus L., A polydactylon L., nom. illeg., Chloris barbata (L.) Nash, hom. illeg., C. dandyana C.D.Adams, C. elata Desv.,C. polydactyla (L.) Sw., C. polydactyla f. pauciradiata Kurtz

Herb.

Departmental distribution: Alto Paraguay, Alto Paraná, Amambay, Boquerón, Central, Concepción, Cordillera, Guairá, Itapúa, Misiones, Paraguarí, Presidente Hayes. Voucher: F. Mereles \& S. Soria 9973 (BM, FCQ, G).

*Tragus australianus S.T.Blake, Univ. Queensl. Papers, Dept. Biol. 1 (18): 12.1941.

Herb. Introduced.

Departmental distribution: Boquerón.

Voucher: F. Mereles \& S. Soria 9942 (FCQ, G).

Trichloris crinita (Lag.) Parodi, Revista Argent. Agron. 14: 63. 1947.

Syn.: Chloris crinita Lag., Chloris mendocina Phil., Chloropsis blanchardiana (E.Fourn. ex Scribn.) Kuntze, Chloropsis crinita (Lag.) Kuntze, Chloropsis mendocina (Phil.) 
Kuntze, Trichloris blanchardiana E.Fourn. ex Scribn., T. crinita (Lag.) Parodi var. triflora Parodi, T. crinita var. typica Parodi, nom. inval., T. mendocina (Phil.) Kurtz, T. mendocina f. blanchardiana (E.Fourn. ex Scribn.) Kurtz, T. mendocina (Phil.) Kurtz ex Seckt, hom. illeg.

Herb.

Departmental distribution: Alto Paraguay, Boquerón.

Voucher: F. Mereles \& S. Soria 9976 (FCQ).

Urochloa brizantha (Hochst. ex A.Rich.) R.D.Webster, Austral. Paniceae (Poaceae) 233. 1987.

Syn.: Brachiaria brizantha (Hochst. ex A.Rich.) Stapf, Panicum brizanthum Hochst. ex A.Rich.

Herb. Introduced.

Departmental distribution: Alto Paraguay, Alto Paraná, Amambay, Canindeyú, Central, San Pedro.

Voucher: F. Mereles et al. 10075 (FCQ).

Urochloa decumbens (Stapf) R.D.Webster, Austral. Paniceae (Poaceae) 234. 1987.

Herb. Introduced.

Departmental distribution: Cordillera, San Pedro.

Voucher: O. Morrone \& J. Pensiero 371 (FCQ).

Urochloa paucispicata (Morong) Morrone \& Zuloaga, Darwiniana 31: 95. 1992.

Syn.: Acroceras paucispicata (Morong) Henrard, Brachiaria paucispicata (Morong) Clayton, Panicum paucispicatum Morong

Herb.

Departmental distribution: Alto Paraguay, Boquerón, Central, Concepción, Cordillera, Presidente Hayes.

Voucher: F. Mereles \& S. Soria 9943 (BM, CTES, FCQ, G).

Urochloa plantaginea (Link.) R.D.Webster, Syst. Bot. 13(4): 607. 1988.

Syn.: Brachiaria plantaginea (Link) Hitchc., Panicum plantagineum Link 
Herb.

Departmental distribution: Alto Paraguay, Central, Concepción, Cordillera, Guairá, San Pedro.

Voucher: J. De Egea et al. 1291 (FCQ).

\section{POLYGONACEAE}

Polygonum acuminatum Kunth, Nov. Gen. Sp. 2: 178. 1817.

Syn.: Persicaria acuminata (Kunth) M.Gómez, Polygonum acuminatum Kunth var. glabrescens Meisn., Polygonum acuminatum var. microstemom Mart. ex Meisn., Polygonum acuminatum var. setigerum (Wedd.) Meisn., Polygonum alfredi Pilg., Polygonum bettfreundianum Lindau, Polygonum floribundum Wedd., Polygonum setigerum Wedd.

Herb.

Departmental distribution: Alto Paraguay, Alto Paraná, Amambay, Boquerón, Caaguazú, Caazapá, Canindeyú, Central, Concepción, Cordillera, Guairá, Itapúa, Misiones, Neembucú, Paraguarí, Presidente Hayes, San Pedro.

Voucher: A. Schinini \& R. Palacios 25915 (FCQ).

Polygonum aviculare L., Sp. Pl. 1: 362 (-363). 1753.

Syn.: Polygonum arenastrum Boreau, P. berteroi Phil., P. striatum K.Koch, P. uruguense H.Gross

Herb. Introduced.

Departmental distribution: Presidente Hayes.

Voucher: T. Rojas 409 (Cialdella and Brandbyge 2001).

Polygonum convolvulus L., Sp. Pl. 1: 364. 1753.

Syn.: Bilderdykia convolvulus (L.) Dumort., Fallopia convolvulus (L.) A.Löve, Tiniaria convolvulus (L.) Webb \& Moq.

Herb. Introduced.

Departmental distribution: Cordillera, Guairá, Itapúa.

Voucher: E. Lurvey 17 (CTES). 
Polygonum hispidum Kunth, Nov. Gen. Sp. (quarto ed.) 2: 178. 1817.

Syn.: Persicaria hispida (Kunth) M.Gómez

Herb.

Departmental distribution: Alto Paraguay, Boquerón, Central, Cordillera, Neembucú, Presidente Hayes.

Voucher: F. Mereles et al. 10117 (FCQ, G).

Polygonum hydropiperoides Michx. var. setaceum (Baldwin) Gleason, Phytologia 4(1): 23. 1952.

Syn.: Polygonum hydropiperoides Michx. var. virgatum (Cham. \& Schltdl.) Meisn., P. setaceum Baldwin, P. virgatum Cham. \& Schltdl.

Herb.

Departmental distribution: Caazapá, Central, Cordillera, Guairá, Itapúa, Misiones, Ñeembucú, Paraguarí, Presidente Hayes, San Pedro.

Voucher: J. De Egea et al. 691 (BM, FCQ).

Polygonum lapathifolium L., Sp. Pl. 1: 360. 1753.

Syn.: Persicaria laphatifolia (L.) Delarbre, Polygonum ferrugineum Wedd. var. patagonicum (Speg.) Macloskie, Polygonum lanigerum R.Br., Polygonum persicaria L. var. vernicosum Cham. \& Schltdl., Polygonum spectabile Mart. ex Meisn. var. patagonica Speg., Polygonum utriculatum J.Remy

Herb. Introduced.

Departmental distribution: Concepción, Presidente Hayes.

Voucher: F. Mereles 1387 (FCQ).

Polygonum meisnerianum Cham. \& Schltdl., Linnaea 3(1): 40-42. 1828.

Syn.: Persicaria meisneriana (Cham. \& Schltdl.) M.Gómez, Polygonum beyrichianum Cham. \& Schltdl., Polygonum chamissoeanum Wedd., Polygonum meisnerianum Cham. \& Schltdl. var. beyrichianum (Cham. \& Schltdl.) Meisn., Polygonum meisnerianum var. setosum Chodat \& Hassl.

Herb. 
Departmental distribution: Alto Paraná, Amambay, Caazapá, Central, Cordillera, Guairá, Itapúa, Misiones, Neembucú, Paraguarí, San Pedro.

Voucher: R. Degen 9 (FCQ).

Polygonum punctatum Elliot, Sketch Bot. S. Carolina 1(5): 455-456. 1821.

Syn.: Persicaria punctata (Elliot) Small, Polygonum acre Kunth, hom. illeg., Polygonum epilobioides Wedd., Polygonum hydropiperoides Pursh, hom. illeg.

Herb.

Departmental distribution: Alto Paraguay, Alto Paraná, Amambay, Caaguazú, Caazapá, Canindeyú, Central, Concepción, Cordillera, Guairá, Itapúa, Misiones, Neembucú, Paraguarí, Presidente Hayes, San Pedro.

Voucher: F. Mereles et al. 10019 (BM, CTES, FCQ, G).

Rumex obovatus Danser, Ned. Kruidk. Arch. 1920: 241. 1921.

Syn.: Rumex paraguayensis auct. non D.Parodi

Herb.

Departmental distribution: Alto Paraná, Central, Guairá, Presidente Hayes.

Voucher: F. Mereles et al. 10092 (FCQ, G).

Rumex paraguayensis D.Parodi, Anales Soc. Ci. Argent. 5: 160. 1878.

Herb.

Departmental distribution: Alto Paraguay, Boquerón, Central, Cordillera, Misiones, Presidente Hayes.

Voucher: R. Spichiger et al. RS2209 (FCQ).

Rumex pulcher L., Sp. Pl. 1: 336. 1753.

Herb. Introduced.

Departmental distribution: Alto Paraná, Central, Cordillera, Misiones.

Voucher: I. Basualdo 1164 (FCQ). 


\section{PORTULACACEAE}

Portulaca cryptopetala Speg., Anales Soc. Ci. Argent. 82: 217. 1916.

Syn.: Portulaca cryptopetala Speg. var. diversifolia (Poelln.) D.Legrand, P. cryptopetala var. legrandii (Poelln.) D.Legrand, P. cryptopetala f. phenopetala Speg., P. diversifolia D.Legrand, nom. illeg., P. diversifolia Poelln. var. legrandii (Poelln.) D.Legrand P. legrandii Poelln.

Herb.

Departmental distribution: Alto Paraguay, Central, Neembucú, Paraguarí, Presidente Hayes, San Pedro.

Voucher: F. Mereles 10125 (FCQ).

Portulaca oleracea L., Sp. Pl. 1: 445. 1753.

Syn.: Portulaca cryptopetala Speg. var. poellnitziana (D.Legrand) D.Legrand, P. marginata Kunth, $P$. neglecta Mack. \& Bush, P. oleracea L. var. granulato-stellulata Poelln., P. oleracea var. macrantha Eggers, P. oleracea var. micrantha Eggers, P. oleracea var. parviflora (Haw.) Griseb., P. oleracea var. silvestris (Montandon) Thell., $P$. poellnitziana D.Legrand, P. retusa Engelm.

Herb. Introduced.

Departmental distribution: Alto Paraguay, Boquerón, Central, Paraguarí, Presidente Hayes, San Pedro.

Voucher: F. Mereles \& S. Soria 9994 (FCQ).

\section{RUBIACEAE}

Borreria eryngioides Cham. \& Schltdl. var. affinis (DC.) K.Schum., Fl. Bras. 6(6): 48. 1888.

Syn.: Borreria affinis DC., B. assurgens (Ruiz \& Pav.) Griseb., B. assurgens Hassl. var. longisepala Hassl., B. chacoensis Hassl., B. chacoensis var. glabrata Hassl., B. eryngioides Cham. \& Schltdl. var. latifolia Hassl., B. parviflora G.Mey. var. scabra Griseb.

Herb or subshrub.

Departmental distribution: Alto Paraguay, Alto Paraná, Caazapá, Central, Guairá, Presidente Hayes.

Voucher: F. Mereles \& S. Soria 10204 (FCQ, G). 
Borreria verticillata (L.) G.Mey., Prim. Fl. Esseq. 83. 1818.

Syn.: Borreria capitata (Ruiz \& Pav.) DC. f. ferruginea auct. non (A.St.-Hil.) Steyerm., Spermacoce verticillata $\mathrm{L}$.

Herb or subshrub.

Departmental distribution: Alto Paraná, Amambay, Caaguazú, Caazapá, Canindeyú, Central, Concepción, Cordillera, Guairá, Misiones, Neembucú, Paraguarí, Presidente Hayes, San Pedro.

Voucher: J. De Egea et al. 1242 (BM, FCQ).

Mitracarpus megapotamicus (Spreng.) Kuntze, Publ. Field Mus. Nat. Hist., Bot. Ser. 7:331. 1931.

Syn.: Mitracarpium cuspidatum DC., Mitracarpus megapotamicus (Spreng.) Standl., comb. superfl., Mitracarpium peladilla Griseb., Mitracarpus sellowianum Cham. \& Schltdl., Spermacoce megapotamica Spreng.

Herb.

Departmental distribution: Alto Paraguay, Amambay, Boquerón, Caaguazú, Caazapá, Central, Concepción, Guairá, Misiones, Paraguarí, Presidente Hayes.

Voucher: F. Mereles et al. 10021 (FCQ).

Mitracarpus villosus (Sw.) DC., Prodr. 4: 572. 1830.

Syn.: Mitracarpus hirtus auct. non (L.) DC., Spermacoce hirta auct. non L., S. villosa Sw.

Herb.

Departmental distribution: Caaguazú, Caazapá, Canindeyú, Central, Paraguarí. Voucher: F. Mereles et al. 10103 (FCQ).

*Oldenlandia lancifolia (Schumach.) DC., Prodr. 4: 425. 1830.

Syn.: Hedyotis lancifolia Schumach.

Herb. Introduced.

Departmental distribution: Central, Cordillera.

Voucher: F. Mereles 10120 (FCQ). 
Richardia brasiliensis Gomes, Mem. sobre Ipecac. 31. 1801.

Syn.: Richardsonia brasiliensis (Gomes) Hayne, Richardia pilosa auct. non Ruiz \& Pav., Richardsonia rosea A.St.-Hil., Richardia rosea (A.St.-Hil.) Schult.f., Richardia scabra auct. non L., Richardsonia brasiliensis (Gomes) Hayne var. dubia Beauverd \& Felipp., Richardsonia scabra auct. non (L.) A.St.-Hil.

Herb.

Departmental distribution: Alto Paraguay, Alto Paraná, Amambay, Boquerón, Caaguazú, Caazapá, Canindeyú, Central, Concepción, Cordillera, Guairá, Itapúa, Neembucú, Paraguarí, Presidente Hayes, San Pedro.

Voucher: J. De Egea et al. 1234 (FCQ).

Staelia virgata (Link ex Roem. \& Schult.) K.Schum. var. virgata, Fl. Bras. 6(6): 76. 1888.

Syn.: Borreria finitima S.Moore, Mitracarpus virgatus (Link ex Roem. \& Schult.) Cham. \& Schltdl., Spermacoce virgata Link ex Roem. \& Schult., Staelia caespitosa Griseb.

Herb.

Departmental distribution: Alto Paraguay, Concepción, Cordillera, Misiones, Neembucú, Paraguarí, Presidente Hayes.

Voucher: F. Mereles \& J. De Egea 10152 (FCQ, G).

\section{SOLANACEAE}

Nicotiana glauca Graham, Edinburgh New Philos. J. 5: 175. 1828.

Syn.: Acnistus virgatus Griseb., Nicotiana glauca Graham var. angustifolia Comes, Nicotiana glauca var. decurrens Comes, Nicotiana glauca var. grandiflora Comes, Nicotiana glauca f. lateritia Lillo, Nicotiana glauca var. typica Millán, nom. inval., Nicotidendron glauca (Graham) Griseb., Siphaulax glabra Raf.

Shrub or subshrub.

Departmental distribution: Alto Paraguay, Boquerón, Central, Cordillera, Neembucú, Paraguarí.

Voucher: F. Mereles et al. 10156 (BM, FCQ, G). 
Nicotiana longiflora Cav., Descr. Pl. 106. 1802.

Syn.: Nicotiana acuta Griseb., N. acutiflora A.St.-Hil., N. longiflora Cav. var. acutiflora (A.St.-Hil.) Comes, $N$. longiflora var. breviflora Comes, nom. inval., $N$. longiflora var. grandifolia Morong

Herb.

Departmental distribution: Boquerón, Central, Concepción, Cordillera, Paraguarí, Presidente Hayes, San Pedro.

Voucher: F. Mereles et al. 10055 (FCQ).

Physalis angulata L., Sp. Pl. 1: 183. 1753.

Herb.

Departmental distribution: Alto Paraguay, Amambay, Boquerón, Guairá, Presidente Hayes, San Pedro.

Voucher: J. De Egea et al. 1319 (BM, FCQ).

Solanum americanum Mill., Gard. Dict. (ed. 8) no. 5. 1768.

Syn.: Solanum adventitium Polg., S. americanum Mill. var. nodiflorum (Jacq.) Edmonds, S. sciaphilum Bitter, S. curtipes Bitter, S. nigrum L. var. americanum (Mill.) O.E.Schulz, $S$. nigrum var. virginicum L., S. nodiflorum Jacq., S. nodiflorum var. acuminatum Chodat, S. nodiflorum var. micropyllum Hassl., S. nodiflorum var. petiolastrum Dunal, S. nodiflorum var. sapucayense Chodat, S. oleraceum Dunal, S. tenellum Bitter.

Herb or subshrub.

Departmental distribution: Alto Paraguay, Alto Paraná, Amambay, Boquerón, Caaguazú, Caazapá, Canindeyú, Central, Concepción, Cordillera, Guairá, Itapúa, Misiones, Neembucú, Paraguarí, Presidente Hayes, San Pedro.

Voucher: J. De Egea et al. 1320 (BM, FCQ).

Solanum chacoense Bitter, Repert. Spec. Nov. Regni Veg. 11: 18. 1912.

Syn.: Solanum arnezii Cárdenas, S. bitteri Hassl., S. boergeri Bukasov, S. caipipendense Cárdenas, S. calvescens Bitter, S. chacoense var. angustisectum (Hassl.) Hassl., S. chacoense f. caipipendense (Cárdenas) Correll, S. chacoense f. gibberulosum (Juz. \& Bukasov) Correll, S. chacoense f. glabrescens (Hassl.) Hassl., S. chacoense var. latisectum (Hassl.) Hassl., S. chacoense subsp. muelleri (Bitter) Hawkes, S. chacoense f. plurijugum Hassl., S. chacoense f. puberulum Hassl., S. chacoense subsp. subtilius (Bitter) Hawkes, 
S. commersonii var. glabriusculum J.Rémy, S. cuevoanum Cárdenas, S. dolichostigma Buk. ex Lechn., S. emmeae Juz. \& Bukasov, S. garciae Juz. \& Bukasov, S. gibberulosum Juz. \& Bukasov, S. guaraniticum Hassl., S. guaraniticum var. angustisectum Hassl., S. guaraniticum f. glabrescens Hassl., S. guaraniticum var. latisectum Hassl., S. horovitzii Bukasov, S. horovitzii var. glabristylum Hawkes, S. horovitzii var. multijugum Hawkes, S. jamesii var. grandifrons Bitter, S. jujuyense Hawkes, S. knappei Juz. \& Bukasov, S. laplaticum Bukasov, S. limense Correll, S. muelleri Bitter, S. muelleri f. densipilosum Correll, S. parodii Juz. \& Bukasov, S. pseudomaglia L.Planch., S. saltense Hawkes, S. schickii Juz. \& Bukasov, S. subtilius Bitter, S. tuberosum subsp. yanacochense Ochoa, $S$. yanacochense (Ochoa) Gorbat., S. yungasense Hawkes.

Herb.

Departmental distribution: Boquerón, Central, Concepción, Cordillera, Guairá, Itapúa, Paraguarí, Presidente Hayes, San Pedro.

Voucher: I. Basualdo 270 (FCQ).

Solanum granuloso-leprosum Dunal, Prodr. 13(1): 115. 1852.

Syn.: Solanum auriculatum auct. non Aiton, S. verbascifolium f. eupulverulentum Hassl., S. verbascifolium L. f. granuloso-leprosum (Dunal) Hassl.

Shrub or tree.

Departmental distribution: Alto Paraguay, Alto Paraná, Amambay, Caaguazú, Caazapá, Canindeyú, Central, Concepción, Cordillera, Guairá, Itapúa, Misiones, Neembucú, Paraguarí, Presidente Hayes, San Pedro.

Voucher: J. De Egea et al. 1310 (FCQ).

Solanum guaraniticum A.St.-Hil., Mém. Mus. Hist. Nat. 12: 321. 1825.

Syn.: Solanum bonariense L. f. intermedium Hassl., S. bonariense f. leptophyllum Chodat, S. bonariense f. lobatum (Chodat) Hassl., S. bonariense var. paraguariense (Chodat) Chodat, S. bonariense f. subintegrum (Hassl.) Chodat, nom. illeg., S. bonariense f. typicum Hassl., nom. inval., S. bonariense var. villaricense (Morong) Chodat, S. fastigiatum Willd. var. acicularium Dunal, S. paraguariense Chodat, S. villaricense Morong, S. villaricense f. lobatum Hassl., S. villaricense var. paraguariense (Chodat) Hassl., S. villaricense f. subintegrum Hassl., S. villaricense Hassl., nom. inval. var. typicum.

Shrub.

Departmental distribution: Alto Paraná, Caaguazú, Caazapá, Canindeyú, Central, Guairá, Itapúa, Misiones, Paraguarí, Presidente Hayes.

Voucher: F. Mereles et al. 10024 (BM, FCQ, G). 
Solanum palinacanthum Dunal, Prodr. 13(1): 245. 1852.

Syn.: Solanum claviceps Griseb., S. palinacanthum Dunal var. acutilobum Dunal, S. palinacanthum $\mathrm{f}$. velutinum Chodat, S. vexans S.Moore

Shrub or small tree.

Departmental distribution: Alto Paraguay, Alto Paraná, Amambay, Caaguazú, Caazapá, Canindeyú, Central, Concepción, Cordillera, Guairá, Itapúa, Misiones, Neembucú, Paraguarí, Presidente Hayes, San Pedro.

Voucher: F. Mereles et al. 10014 (BM, FCQ, G).

Solanum pseudocapsicum L., Sp. Pl. 1: 184. 1753.

Syn.: Solanum capsicastrum Link ex Schauer, S. capsicastrum var. cadguazuense Chodat, S. diflorum Vell., S. diflorum var. angustifolium Kuntze, S. ipecacuanha Chodat var. calvescens Chodat, S. pavimenti L.B.Sm. \& Downs, S. pseudocapsicum var. ambiguum Hassl., S. pseudocapsicum L. f. calvescens (Chodat) Hassl., S. pseudocapsicum ssp. diflorum (Vell.) Hassl., S. pseudocapsicum var. hygrophilum (Schltdl.) Hassl., S. pseudocapsicum var. normale Kuntze, $S$. pseudocapsicum var. parvifolium Kuntze, $S$. pseudocapsicum f. pilosum Kuntze, S. pseudocapsicum f. pilosulum Hassl., S. pseudocapsicum var. sendtnerianum Hassl., S. pseudocapsicum var. typicum Hassl., nom. inval.

Subshrub.

Departmental distribution: Alto Paraguay, Amambay, Caaguazú, Caazapá, Canindeyú, Central, Cordillera, Guairá, Misiones, Neembucú, Paraguarí, Presidente Hayes, San Pedro.

Voucher: J. De Egea et al. 1263 (FCQ).

Solanum sisymbriifolium Lam., Tabl. Encycl. 2: 25. 1794.

Syn.: Solanum balbisii Dunal, S. balbisii var. bipinnata Hook., S. balbisii var. oligospermum Sendtn., S. balbisii var. purpureum Hook., S. bipinnatifidum Larrañaga, S. decurrens Balb., S. sisymbriifolium f. albiflorum Kuntze, S. sisymbriifolium Lam. var. brevilobum Dunal, S. sisymbriifolium var. heracleifolium Sendtn., nom. inval., S. sisymbriifolium f. lilacinum Kuntze, S. sisymbriifolium var. macrocarpum Kuntze, S. sisymbriifolium var. oligospermum (Sendtn.) Dunal, S. sisymbriifolium var. purpureiflorum Dunal, S. subviscidum Schrank

Herb or subshrub.

Departmental distribution: Alto Paraguay, Boquerón, Caaguazú, Central, Cordillera, Guairá, Itapúa, Neembucú, Paraguarí, Presidente Hayes, San Pedro.

Voucher: F. Mereles et al. 10015 (FCQ, G). 
Solanum tweedieanum Hook., Bot. Mag. 62: t.3385. 1835.

Syn.: Solanum atriplicoides Herter, S. meizonanthum Bitter, S. physalidicalyx Bitter, S. physalidicalyx var. integrescens Bitter, S. physalidicalyx var. plurilobulatum Bitter, S. sidifolium Speg.

Herb.

Departmental distribution: Alto Paraguay, Boquerón, Presidente Hayes.

Voucher: F. Mereles \& S. Soria 9992 (FCQ, G, BM, CTES).

Solanum viarum Dunal, Prodr. 13(1): 240. 1852.

Syn.: Solanum chloranthum DC., hom. illeg., S. reflexum auct. non Schrank, S. viridiflorum Schltdl.

Shrub.

Departmental distribution: Alto Paraguay, Alto Paraná, Boquerón, Caazapá, Canindeyú, Central, Concepción, Cordillera, Guairá, Neembucú, Paraguarí, San Pedro. Voucher: F. Mereles \& S. Soria 9974 (FCQ).

\section{TALINACEAE}

Talinum fruticosum (L.) Juss., Gen. Pl. 312. 1789.

Syn.: Portulaca fruticosa L., P. racemosa L., P. triangularis Jacq., Talinum racemosum (L.) Rohrb., T. triangulare (Jacq.) Willd.

Subshrub. Introduced.

Departmental distribution: Alto Paraguay, Boquerón, Cordillera, Paraguarí, Presidente Hayes, San Pedro.

Voucher: F. Mereles \& S. Soria 9963 (BM, CTES, FCQ, G).

Talinum paniculatum (Jacq.) Gaertn., Fruct. Sem. Pl. 2: 219. 1791.

Syn.: Portulaca paniculata Jacq., P. patens L., Talinum patens (L.) Willd.

Herb. Introduced.

Departmental distribution: Alto Paraguay, Amambay, Boquerón, Caazapá, Canindeyú, Central, Cordillera, Guairá, Paraguarí, Presidente Hayes, San Pedro.

Voucher: M. Peña-Chocarro \&J. De Egea 2453 (BM, CTES, FCQ, MO). 


\section{URTICACEAE}

Parietaria debilis G.Forst., Fl. Ins. Austr. 73. 1786.

Syn.: Freirea erecta Phil., F. humifusa Gay, Parietaria debilis G.Forst. var. diffusa Wedd., $P$. debilis var. gracilis (Lowe) Wedd., $P$. debilis var. micrantha (Ledeb.) Wedd., $P$. fernandeziana (Steud.) L.E.Navas, P. gracilis Lowe, P. humifusa (Gay) Blume, P. micrantha Ledeb., Urtica fernandeziana Steud., U. parietariaefolia Bertero ex Steud.

Herb.

Departmental distribution: Alto Paraguay, Alto Paraná, Amambay, Caazapá, Central, Concepción, Cordillera, Guairá, Paraguarí.

Voucher: J. De Egea 1295 (BM, FCQ, G).

\section{VERBENACEAE}

Aloysia virgata (Ruiz \& Pav.) Pers. var. platyphylla (Briq.) Moldenke, Phytologia 2: 310. 1947.

Syn.: Aloysia naviculata Ravenna, A. virgata (Ruiz \& Pav.) Pers. var. elliptica (Briq.) Moldenke, Lippia virgata (Ruiz \& Pav.) Steud. var. elliptica Briq., L. virgata var. platyphylla Briq.

Shrub.

Departmental distribution: Alto Paraguay, Alto Paraná, Amambay, Central, Concepción, Cordillera, Guairá, Itapúa, Misiones, Neembucú, Paraguarí, Presidente Hayes, San Pedro.

Voucher: F. Mereles 9928 (BM, FCQ, G).

*Glandularia cabrerae (Moldenke) Botta, Bol. Soc. Argent. Bot. 26: 243. 1990.

Syn.: Verbena cabrerae Moldenke, $V$. dissecta Willd. ex Spreng. f. alba Moldenke

Herb.

Departmental distribution: Alto Paraguay, Alto Paraná.

Voucher: F. Mereles et al. 10067 (FCQ).

Glandularia tomophylla (Briq.) P.Peralta, Darwiniana 45: 241. 2007.

Syn.: Glandularia spectabilis (Moldenke) Botta, Verbena calliantha Briq., V. calliantha var. microsoma Briq., V. megapotamica Spreng. var. pinnatiloba Kuntze, V. pin- 
natiloba (Kuntze) Moldenke, V. ramboi Moldenke, $V$. spectabilis Moldenke, $V$. storeoclada Briq., $V$. tomophylla Briq.

Herb.

Departmental distribution: Alto Paraguay, Alto Paraná, Caaguazú, Central, Guairá, Itapúa, Misiones, Neembucú, Paraguarí.

Voucher: J. De Egea et al. 1136 (BM, FCQ, G).

Glandularia tweediana (Niven ex Hook.) P.Peralta, Ann. Missouri Bot. Gard. 98(3): 400. 2011.

Syn.: Glandularia incisa (Hook.) Tronc., Verbena incisa Hook., V. megapotamica Spreng. var. truncatula Briq., V. megapotamica var. tweediana (Niven ex Hook.) Kuntze, $V$. tweediana Niven ex Hook., V. tweedieana var. arriana Niven ex Maund.

Herb.

Departmental distribution: Alto Paraguay, Caaguazú, Central, Cordillera, Guairá, Neembucú, Paraguarí, Presidente Hayes, San Pedro.

Voucher: J. De Egea et al. 1274 (BM, FCQ).

Stachytarpheta cayennensis (Rich.) Vahl, Enum. Pl. 1: 208. 1804.

Syn.: Stachytarpheta australis Moldenke, S. cayennensis (Rich.) Vahl f. alba Moldenke ex Tronc. \& Botta, S. cayennensis f. albiflora Moldenke, S. cayennensis var. candicans Briq., S. cayennensis var. virescens Briq., S. dichotoma (Ruiz \& Pav.) Vahl, S. maximiliani Schauer var. ciliaris Moldenke, S. patens Moldenke, Verbena cayennensis Rich., V. dichotoma Ruiz \& Pav.

Herb or subshrub.

Departmental distribution: Alto Paraguay, Alto Paraná, Amambay, Caaguazú, Caazapá, Canindeyú, Central, Concepción, Cordillera, Guairá, Itapúa, Misiones, Neembucú, Paraguarí, Presidente Hayes, San Pedro.

Voucher: J. De Egea et al. 1221 (BM, FCQ).

Verbena litoralis Kunth var. litoralis, Nov. Gen. Sp. 2: 276, t. 137. 1818.

Syn.: Verbena affinis M.Martens \& Galeotti, V. approximata Briq., V. bonariensis L. var. litoralis (Kunth) Gillies \& Hook. ex Hook., V. caracasana Kunth, V. cordobensis Briq., V. integrifolia Sessé \& Moç., V. integrifolia f. albiflora Chodat, V. litoralis Kunth var. albiflora Moldenke, $V$. litoralis f. albiflora (Moldenke) Moldenke $V$. litoralis $\mathrm{f}$. angustifolia Chodat, $V$. litoralis var. caracasana (Kunth) Briq., V. litoralis 
var. glabrior Benth., V. litoralis var. leptostyachya Schauer, V. litoralis var. pycnostachya Schauer, nom. inval., V. nudiflora Nutt. ex Turcz.

Herb.

Departmental distribution: Alto Paraná, Amambay, Caaguazú, Caazapá, Canindeyú, Central, Concepción, Cordillera, Guairá, Itapúa, Misiones, Neembucú, Paraguarí, Presidente Hayes, San Pedro.

Voucher: F. Mereles et al. 10091 (FCQ, G).

Verbena montevidensis Spreng., Syst. Veg. (ed. 16) 2:747. 1825.

Syn.: Verbena isabellei Briq., V. minutiflora Briq. ex Moldenke, V. minutiflora var. peruviana Moldenke.

Herb.

Departmental distribution: Alto Paraguay, Caaguazú, Caazapá, Central, Cordillera, Guairá, Itapúa, Misiones, Paraguarí, Presidente Hayes.

Voucher: R. Degen et al. 1729 (FCQ).

\section{Acknowledgments}

We thank Monsanto Paraguay, the Instituto de Biotecnología Agrícola (INBIO) and the company Dekalpar S.A. for financing the fieldwork and facilitating access to crop trials. We also thank Silvina Soria and Gerardo Berton for joining fieldtrips and sharing their expertise on crop and weed management. We are especially grateful to the curators of FCQ, BM and CTES for their assistance with specimen identification, and to Mariana Martinez for her kind support.

\section{References}

Bajwa AA (2014) Sustainable weed management in conservation agriculture. Crop Protection 65: 105-113. doi: 10.1016/j.cropro.2014.07.014

Baker HG (1965) Characteristics and modes of origins of weeeds. In: Baker GH, Stebbins GL (Eds) The Genetics of Colonizing Species. Academic, New York, 147-172.

Baker HG (1974) The evolution of weeds. Annual Review of Ecology and Systematics 5: 1-24. doi: 10.1146/annurev.es.05.110174.000245

Base de dados nacional de espécies exóticas invasoras I3N Brasil (2016) Instituto Hórus de Desenvolvimento e Conservação Ambiental, Florianópolis, SC. http://i3n.institutohorus.org. br/www [accessed 14.06.2016] 
Bertoni GT, Gorham R (1973) The Geography of Paraguay. In: Gorham R (Ed.) Paraguay: Ecological Essays. Academy of the Arts and Sciences of the Americas, Miami, 9-32.

Bruinsma J (2009) The resource outlook to 2050: by how much do land, water and crop yields need to increase by 2050? Paper presented at the FAO Expert Meeting, 24-26 June 2009, Rome on "How to Feed the World in 2050". Food and Agriculture Organization of the United Nations, Economic and Social Development Department, Rome, 3-6.

Cialdella AM, Brandbyge J (2001) Poligonaceae. Flora del Paraguay 33: 38-39.

Daehler CC (1998) The taxonomic distribution of invasive angiosperm plants: ecological insights and comparison to agricultural weeds. Biological Conservation 84: 167-180. doi: 10.1016/S0006-3207(97)00096-7

De Egea J, Peña-Chocarro M, Espada C, Knapp S (2012) Checklist of vascular plants of the Department of Neembucú, Paraguay. PhytoKeys 9: 15-179. doi: 10.3897/phytokeys.9.2279

De Egea J, Mereles F, Céspedes G (2016) Tragus australianus (Poaceae, Cynodonteae), nuevo registro de especie exótica para el Paraguay. Bonplandia 25(1): 73-77.

DGEEC (2015) Compendio Estadístico 2014. Dirección General de Estadística, Encuestas y Censos; Secretaría Técnica de Planificación del Desarrollo Económico y Social; Gobierno Nacional, 49-51.

Ferrari G (2012) Malezas y sus dosis de control. Monsanto Argentina S.A.I.C. 247.

Figueroa JA, Castro SA, Marquet PA, Jaksic FM (2004) Exotic plant invasions to the mediterranean region of Chile: causes, history and impacts. Revista Chilena de Historia Natural 77: 465-483. doi: 10.4067/S0716-078X2004000300006

González Erico E (2007) Suelos del Paraguay. In: Salas-Dueñas D, Facetti JF (Eds) Biodiversidad del Paraguay: una aproximación a sus realidades. Fundación Moisés Bertoni para la Conservación de la Naturaleza, Agencia Internacional de Desarrollo de los Estados Unidos de América (USAID) y el Global Environment Facility, Banco Mundial (GEF/BM), 47-56.

Guglieri-Caporal A, Machado Caporal FJ, Leite Kufner DC, de Matos Alves F (2011) Flora invasora de cultivos de aveia-preta, milho e sorgo em região de cerrado do Estado de Mato Grosso do Sul, Brasil. Bragantia, Campinas 70(2): 247-254.

Heap I (2016) The International Survey of Herbicide Resistant Weeds. http://www.weedscience.org [accesed 16.01.2016]

Hill MO, Roy D, Thompson K (2002) Hemeroby, urbanity and ruderality: bioindicators of disturbance and human impact. Journal of Applied Ecology 39: 708-720. doi: 10.1046/j.1365-2664.2002.00746.x

Kuester A, Conner JK, Culley T, Baucom RS (2014) How weeds emerge: a taxonomic and trait-based examination using United States data. New Phytologist 202: 1055-1068. doi: $10.1111 /$ nph. 12698

Lorenzi H (2000) Plantas daninhas do Brasil: terrestres, aquáticas, parasitas e tóxicas ( ${ }^{\text {rd }}$ edn). Instituto Plantarum, Nova Odessa, 608 pp.

Lurvey EL (1983) Malezas de algodonales, maizales y otros cultivos anuales. Ministerio de Agricultura y Ganadería, Cuerpo de Paz de los EE.UU., Asunción 203, 11 pp.

Mereles F, Degen R (1994) Contribución al estudio de la flora y vegetación del Chaco Boreal Paraguayo. Rojasiana 2(1): 36-38. 
Pott A, Pott VJ, de Souza TW (2006) Plantas daninhas de pastagem na Região dos Cerrados. Embrapa Gado de Corte, Corumbá, 336 pp.

Proyecto Sistema Ambiental del Chaco: suelos del Chaco (1992-1997) Informe Técnico.

Dirección de Ordenamiento Ambiental, Sub-Secretaría de Recursos Naturales y Medio Ambiente, Ministerio de Agricultura y Ganadería, Paraguay (DOA-SSERNMA-MAG) e Instituto Federal de Geociencias y Recursos Naturales, Alemania (BGR).

Pyšek P, Richardson DM, Rejmánek M, Webster GL, Williamson M, Kirschner J (2004) Alien plants in checklists and floras: towards better communication between taxonomists and ecologists. Taxon 53(1): 131-143. doi: 10.2307/4135498

Ramella L, Perret P (Eds) (2008-2014) Colección Flora del Paraguay. Conservatoire et Jardin botaniques de la Ville de Genève \& Missouri Botanical Garden, St Louis.

Rejmánek M, Richardson DM (1996) What attributes make some plan species more invasive? Ecology 77(6): 1655-1661. doi: 10.2307/2265768

Richardson DM, Pyšek P, Rejmánek M, Barbour MG, Panetta FD, West CJ (2000) Naturalization and invasion of alien plants: concepts and definitions. Diversity and Distributions 6: 93-107. doi: 10.1046/j.1472-4642.2000.00083.x

Smith AR, Kreier H, Haufler CH, Korall P, Schneider H, Wolf PG (2006) A classification for extant ferns. Taxon 55: 705-731. doi: 10.2307/25065646

Spichiger R (Ed.) (1987-1989) Colección Flora del Paraguay. Conservatoire et Jardin botaniques de la Ville de Genève \& Missouri Botanical Garden, St Louis.

Spichiger R, Bocquet G (Eds) (1983-1985) Colección Flora del Paraguay. Conservatoire et Jardin botaniques de la Ville de Genève \& Missouri Botanical Garden, St Louis.

Spichiger R, Ramella L (Eds) (1990-2002) Colección Flora del Paraguay. Conservatoire et Jardin botaniques de la Ville de Genève \& Missouri Botanical Garden, St Louis.

Stevens PF (2001) Angiosperm Phylogeny Website. Version 12, July 2012 [continuously updated]. http://www.mobot.org/MOBOT/research/APweb/ [accessed: 14.06.2016]

Thiers B (continuously updated) Index Herbariorum: A global directory of public herbaria and associated staff. New York Botanical Garden's Virtual Herbarium. http://sweetgum.nybg. org/science/ih/ [accessed: 14.06.2016]

TROPICOS Database (2016) Missouri Botanical Garden. http://www.tropicos.org [accessed: 14.06.2016]

WWF (2014) World Wildlife Fund - El Crecimiento de la Soja: Impactos y Soluciones. WWF International, Gland, Suiza, 22-24.

WWF (2016) World Wildlife Fund - Análisis social, económico y ambiental de la producción de soja y carne en Paraguay. WWF-Paraguay y Federal Ministry for the Environment, Nature Conservation and Nuclear Safety, Federal Republic of Germany. Asunción, Paraguay, 16-18. Zuloaga FO, Belgrano MJ (Eds) (2015) Catálogo de las Plantas Vasculares del Cono Sur. http://www.darwin.edu.ar/Proyectos/FloraArgentina/FA.htm [accesed: 22.07.2015] Zuloaga FO, Morrone O, Pensiero JF (2014) Gramineae VI. Flora del Paraguay 45: 338-343. Zuloaga FO, Morrone O, Rúgolo De Agrasar ZE, Anton AM, Arriaga MO, Cialdella AM (1994) Gramineae V. Flora del Paraguay 23: 148-149. 